\title{
Regular Polytopes of Nearly Full Rank
}

\author{
Peter McMullen
}

Received: 8 June 2010 / Revised: 10 February 2011 / Accepted: 17 February 2011 /

Published online: 9 March 2011

(C) Springer Science+Business Media, LLC 2011

\begin{abstract}
An abstract regular polytope $\mathcal{P}$ of rank $n$ can only be realized faithfully in Euclidean space $\mathbb{E}^{d}$ of dimension $d$ if $d \geq n$ when $\mathcal{P}$ is finite, or $d \geq n-1$ when $\mathcal{P}$ is infinite (that is, $\mathcal{P}$ is an apeirotope). In case of equality, the realization $P$ of $\mathcal{P}$ is said to be of full rank. If there is a faithful realization $P$ of $\mathcal{P}$ of dimension $d=n+1$ or $d=n$ (as $\mathcal{P}$ is finite or not), then $P$ is said to be of nearly full rank. In previous papers, all the at most four-dimensional regular polytopes and apeirotopes of nearly full rank have been classified. This paper classifies the regular polytopes and apeirotopes of nearly full rank in all higher dimensions.
\end{abstract}

Keywords Abstract regular polytope - Realization · Faithful · Nearly full rank · Fine Schläfli symbol

\section{Introduction}

While the finite regular polyhedra and honeycomb of cubes in ordinary threedimensional space have long been known, various generalizations of them-which are also regular in a similar geometric sense-occur only considerably more recently. Petrie and Coxeter [3] described the sponges which bear their name, Grünbaum [13] found all but one of the remaining regular apeirohedra in $\mathbb{E}^{3}$, while Dress $[8,9]$ found the missing example and proved that the classification was then complete. In [23] (see also [24, Sects. 7E, 7F] - this monograph is a general reference to what was known up to 2002), the present author and Schulte classified completely all the faithfully realized regular polytopes and discrete regular apeirotopes in dimensions up to three, including a quick proof of Dress's result. This author then successively classified in [16] the regular polytopes and apeirotopes of full rank in each higher dimension

P. McMullen ( $\otimes)$

University College London, Gower Street, London WC1E 6BT, England, UK

e-mail: p.mcmullen@ucl.ac.uk 
(the terms used here will be defined later), in [17] the four-dimensional (finite) regular polyhedra (those with planar faces can be recovered from [1, 2]), and in [18] the four-dimensional regular apeirotopes of rank 4 . The polytopes of the latter two papers are of nearly full rank; in this paper, we classify such regular polytopes and apeirotopes in dimension at least five.

Let us briefly outline the contents of the paper. In Sects. 2 and 3 we describe those parts of the general theory of regular polytopes and their realizations which we need. In Sect. 4 we discuss notation, and in Sect. 5 we recall various operations which lead from one regular polytope or apeirotope to another. Certain of the polytopes are blended and so arise from lower-dimensional ones; we treat these in Sect. 6. The vertex-figure of a polytope (or apeirotope) of nearly full rank is itself of full or nearly full rank; in Sect. 7 we treat the former possibility and generalize results which have been obtained previously in lower dimensions. Section 8 imposes some important further restrictions in the 'gateway' dimension 5. In Sect. 9 we describe two constructions which lead from finite regular polytopes to regular apeirotopes, obtaining results analogous to those of [18]. In several earlier papers, we have used a certain mixing operation which is, in a sense, a dual of the Petrie operation; in Sect. 10 we generalize this operation, which is called Petrie contraction. Section 11 introduces a family of polytopes and apeirotopes which build on Petrie contraction and turn out to be closely related to the Gosset polytopes. In Sect. 12, we apply twisting operations to derive some regular polytopes and apeirotopes from Coxeter groups; we also show that many polytopes of the previous sections can be obtained by a variant of twisting. Section 13 then treats further polytopes obtained by twisting, again related to Gosset polytopes. In lower dimensions, there are regular polytopes and apeirotopes whose symmetry groups consist of direct isometries alone; in Sect. 14, we show that there is one solitary extra example of such in $\mathbb{E}^{5}$, and no more in higher dimensions. Finally, in Sect. 15 we conclude the paper with some comments about possible future directions for this line of research.

For the general background on abstract regular polytopes, we refer the reader to the monograph [24]; for the most part, we shall not cite original papers on the theory of abstract regular polytopes directly. In this paper, we concentrate almost entirely on the geometric aspects of the theory, that is, on realized regular polytopes. In some respects, particularly in the abstract theory, we have changed notation from that used hitherto. The motivation for this is to introduce a greater degree of consistency. It is hoped that the reader familiar with earlier work (especially [24]) will not find these changes confusing.

\section{Abstract Regular Polytopes}

We briefly review what we need of the abstract theory. An abstract regular polytope $\mathcal{P}$ of rank $n$ (or $n$-polytope for short) is identified with its automorphism group $\boldsymbol{G}=$ $\left\langle\boldsymbol{r}_{0}, \ldots, \boldsymbol{r}_{n-1}\right\rangle$. Here, the canonical generators $\boldsymbol{r}_{j}$ are involutory, $\boldsymbol{r}_{j}$ and $\boldsymbol{r}_{k}$ commute if $|j-k| \geq 2$, and they satisfy the intersection property

$$
\left\langle\boldsymbol{r}_{i} \mid i \in \mathrm{J}\right\rangle \cap\left\langle\boldsymbol{r}_{i} \mid i \in \mathrm{K}\right\rangle=\left\langle\boldsymbol{r}_{i} \mid i \in \mathrm{J} \cap \mathrm{K}\right\rangle
$$


for each $\mathrm{J}, \mathrm{K} \subseteq \mathrm{N}:=\{0, \ldots, n-1\}$; this makes $\boldsymbol{G}$ a string $C$-group. If we define $\boldsymbol{G}_{j}:=\left\langle\boldsymbol{r}_{i} \mid i \neq j\right\rangle$ for each $j \in \mathrm{N}$, then the initial $j$-face $\mathcal{F}_{j}$ of $\mathcal{P}$ is (identified with) $\boldsymbol{G}_{j}$, and the general $\boldsymbol{j}$-face is a right coset $\boldsymbol{G}_{j} \boldsymbol{s}$ of $\boldsymbol{G}_{j}$ (with $\boldsymbol{s} \in \boldsymbol{G}$ ). Finally, two faces are incident if they meet (as cosets). In fact, we have a partial ordering of $\mathcal{P}$ given by $\boldsymbol{G}_{j} \boldsymbol{s} \leq \boldsymbol{G}_{k} \boldsymbol{t}$ when $j \leq k$ and $\boldsymbol{G}_{j} \boldsymbol{s} \cap \boldsymbol{G}_{k} \boldsymbol{t} \neq \emptyset$; formally, we adjoin two copies of $\boldsymbol{G}$ itself as the unique (-1)- and $n$-faces of $\mathcal{P}$, thus making $\mathcal{P}$ a poset with (unique) minimum and maximum elements.

Remark 2.1 While polytope is the generic term, we often use the term apeirotope for an infinite polytope.

If we let $p_{j}$ be the period of $\boldsymbol{r}_{j-1} \boldsymbol{r}_{j}$ for $j=1, \ldots, n-1$, then $\left\{p_{1}, \ldots, p_{n-1}\right\}$ is called the Schläfli type of $\mathcal{P}$. In general, we can allow some $p_{j}=\infty$; however, in the present context, this may only happen for $j=1$.

Remark 2.2 The use of heavy braces denotes abstract regular polytopes, to distinguish them from geometric ones; subsequently, we shall introduce modifications of the notation. In this context, the entries are always integers or $\infty$, because they are periods of group elements.

For each $0 \leq k<m \leq n$, the subgroup $\left\langle\boldsymbol{r}_{k}, \ldots, \boldsymbol{r}_{m-1}\right\rangle$ is also a string C-group; the corresponding regular $(m-k)$-polytope is called the (initial) $(k, m)$-section of $\mathcal{P}$ (thus the $(0, n)$-section is $\mathcal{P}$ itself). There are various special cases. For $k=0$, we have the $m$-face of $\mathcal{P}$, with vertex, edge, ridge and facet the cases $m=0,1, n-3$ and $n-2$, respectively. For $m=n$, we have the $(n-k)$-coface of $\mathcal{P}$, with $k=1$ and 2 giving the vertex-and edge-figure, respectively. (Observe that 'coface' as defined here is to be distinguished from the term 'co-face' of [24, p. 23]; thus $r$-coface means co$(n-r-1)$-face in the earlier terminology, with the prefix now-more conveniently, we feel-denoting the rank.)

Remark 2.3 If we write $\boldsymbol{G}_{r s}:=\boldsymbol{G}_{r} \cap \boldsymbol{G}_{s}$ for $r<s$, then for $k \leq j<m$, the $j$-faces of the initial $(k, m)$-section are $\boldsymbol{G}_{j} \boldsymbol{g}$, with $\boldsymbol{g} \in \boldsymbol{G}_{k-1, m}$; observe that, if $i<k-1$ or $i>m$, then $\boldsymbol{r}_{i}$ fixes all the section.

If $\mathcal{P}$ with group $\left\langle\boldsymbol{r}_{0}, \ldots, \boldsymbol{r}_{n-1}\right\rangle$ and $\mathcal{Q}$ with group $\left\langle\boldsymbol{s}_{0}, \ldots, \boldsymbol{s}_{m-1}\right\rangle$ are two regular polytopes such that there exists a group homomorphism $\Phi$ with

$$
\boldsymbol{r}_{j} \Phi= \begin{cases}\boldsymbol{s}_{j} & \text { if } j \leq m-1 \\ \boldsymbol{e} & \text { if } j \geq m,\end{cases}
$$

then we say that $\mathcal{P}$ covers $\mathcal{Q}$ or that $\mathcal{Q}$ is a quotient of $\mathcal{P}$. Note that we specifically allow $\operatorname{rank} \mathcal{P}>\operatorname{rank} \mathcal{Q}$.

The amalgamation problem asks whether, given two regular $n$-polytopes $\mathcal{P}$ and $\mathcal{Q}$ such that the vertex-figure of $\mathcal{P}$ and the facet of $\mathcal{Q}$ are isomorphic, there exists a regular $(n+1)$-polytope whose facet is $\mathcal{P}$ and vertex-figure is $\mathcal{Q}$. If one such does exist, then there is a universal one, denoted $\{\mathcal{P}, \mathcal{Q}\}$, which covers (as quotients) any other. In terms of the corresponding automorphism groups $\boldsymbol{G}$ and $\boldsymbol{H}$, 
we must think of $\boldsymbol{G}=\left\langle\boldsymbol{r}_{0}, \ldots, \boldsymbol{r}_{n-1}\right\rangle$ and $\boldsymbol{H}=\left\langle\boldsymbol{r}_{1}, \ldots, \boldsymbol{r}_{n}\right\rangle$, so that the group of $\{\mathcal{P}, \mathcal{Q}\}$ is $\left\langle\boldsymbol{r}_{0}, \ldots, \boldsymbol{r}_{n}\right\rangle$, with no relators apart from $(0 n)^{2}$ (which we need to make a string group) other than those arising from $\boldsymbol{G}$ and $\boldsymbol{H}$. A further important question is whether the universal amalgam is finite if $\mathcal{P}$ and $\mathcal{Q}$ are.

These ideas have natural geometric counterparts, where universality often comes into consideration.

\section{Realizations}

There are many candidates for spaces in which regular polytopes $\mathcal{P}$ might be realized geometrically. The usual (and generally most useful) context of realizations is of those in Euclidean spaces, because it is in these that we obtain the richest structure; see [14]. However, it is worth noting that some basic results have a common statement if they are posed for realizations in spherical or hyperbolic spaces as well (see particularly [16, Sect. 3]); we shall occasionally adopt this approach here.

In this geometric context, we have a representation of $\boldsymbol{G}$ as a homomorphic image $\mathcal{G}=\boldsymbol{G} \Phi$ in the group $\mathcal{I}=\mathcal{I}(\mathbb{E})$ of isometries of some Euclidean space $\mathbb{E}$. Then $\mathcal{G}=\left\langle R_{0}, \ldots, R_{n-1}\right\rangle$, where each $R_{j}=\boldsymbol{r}_{j} \Phi$ is a reflexion (that is, involutory) or the identity $I$, which we often identify with its mirror of fixed points

$$
\left\{x \mid x R_{j}=x\right\} .
$$

The structure of the corresponding realization $P$ of the abstract polytope $\mathcal{P}$ is given by Wythoff's construction. We identify (geometric) faces with their vertex-sets, bearing in mind the partial order induced from the abstract polytope. There is an initial vertex $v \in W:=R_{1} \cap \cdots \cap R_{n-1}$, the Wythoff space of the realization. The vertex-set of the initial $j$-face is vert $F_{j}:=v \mathcal{G}_{j}$, where $\mathcal{G}_{j}=\boldsymbol{G}_{j} \Phi$ for $j=1, \ldots, n-1$; all other (proper) faces are images of the initial faces under $\mathcal{G}$. Thus vert $P=v \mathcal{G}$. It is also convenient to define $F_{-1}:=\emptyset$ and $F_{n}:=P$ : thus, $F_{-1} \subseteq F_{0} \subseteq \cdots \subseteq F_{n}$. The (affine) subspace $\mathbb{E}:=$ aff vert $P$ spanned by vert $P$ is called the ambient space of $P$; we lose no generality in thinking of this as the whole space in which we work. We then write $\operatorname{dim} P:=\operatorname{dim} \mathbb{E}$, which is the dimension of $P$.

The realization $P$ of $\mathcal{P}$ is faithful if the representation is an isomorphism of groups and the abstract and geometric posets are isomorphic.

By definition, $\mathcal{G}$ yields the initial faces of $P$; in particular, $F_{n-1}$ is the facet of $\mathcal{P}$; its symmetry group is $\mathcal{G}_{n-1}:=\left\langle R_{0}, \ldots, R_{n-2}\right\rangle$. There is no harm in adopting the intuitive view-point that an edge is the (line-)segment joining its two vertices, and that a 2 -face is the polygon determined by its constituent (vertices and) edges. If we write $w$ for the mid-point of the edge $\left\{v, v R_{0}\right\}$, then $w$ is the initial vertex of the vertex-figure of $\mathcal{P}$, whose symmetry group is $\mathcal{G}_{0}=\left\langle R_{1}, \ldots, R_{n-1}\right\rangle$.

We often find it more convenient to use $v R_{0}$ rather than $w$ as the initial vertex of the vertex-figure; for most purposes, this makes little difference, since the combinatorics are not altered, and we have the added convenience that the vertices of the vertex-figure occur among those of the polytope itself. In the finite case, we usually suppose that the origin $o$ is the centre of $P$ (that is, the centroid of its vertices); thus, 
$\mathcal{G}$ is an orthogonal group. When we are dealing with apeirotopes, it is usual to take the initial vertex $v=F_{0}$ to be $o$, so that now $\mathcal{G}_{0}$ is an orthogonal group.

More generally, if we write $W_{k}:=\bigcap\left\{R_{i} \mid i \neq k\right\}$ and take $v_{k} \in W_{k} \backslash R_{k}$, then $v_{k}$ is the initial vertex of the (initial) $(k, m)$-section, whose vertex-set is $v_{k} \mathcal{G}_{k-1, m}$, with $\mathcal{G}_{r s}:=\boldsymbol{G}_{r s} \Phi$ for each $r$ and $s$. Bear in mind Remark 2.3 here; effectively, of course, the symmetry group of this section is $\left\langle R_{k}, \ldots, R_{m-1}\right\rangle$.

Remark 3.1 If we drop the intersection property (2.1), then we obtain a regular prepolytope (geometric or abstract, as appropriate). Pre-polytopes occur quite naturally in the context of realizations, in which case we often use the terms degenerate or non-polytopal.

There are important restrictions on faithful realizations, which we state in general terms; we refer to $[24$, Sects. 5B, 5C] for proofs.

Theorem 3.2 Let $P$ be a faithful realization of a finite abstract regular polytope $\mathcal{P}$, whose ambient space is Euclidean. Then $\operatorname{dim} P \geq \operatorname{rank} \mathcal{P}$.

Theorem 3.3 Let $P$ be a faithful realization of an abstract regular apeirotope $\mathcal{P}$, whose ambient space is Euclidean. Then $\operatorname{dim} P \geq \operatorname{rank} \mathcal{P}-1$.

A unified picture of these two results is obtained by taking the ambient space of a finite regular polytope to be a sphere, whose dimension is one less than that of the corresponding Euclidean space. See [16] for further details on this.

The difference $\operatorname{dim} P-\operatorname{rank} P$ in the finite case, or $\operatorname{dim} P-\operatorname{rank} P+1$ for an apeirotope, will be called the rank deficit of $P$, denoted rankdef $P$. Then $P$ is of full rank if rankdef $P=0$. In this paper, we are classifying polytopes $P$ of nearly full rank, meaning that rankdef $P=1$.

We shall call the sequence $\left(\operatorname{dim} R_{0}, \operatorname{dim} R_{1}, \ldots, \operatorname{dim} R_{n-1}\right)$ the mirror vector of the realization (in previous works, we have used the less evocative term dimension vector - the new name was suggested by Pellicer and Schulte [25]). In lower dimensions $d(\leq 4)$ an important first step in the classification of the faithfully realized regular polytopes of a fixed rank $n$ in a fixed space was to determine which mirror vectors could occur. We have already solved this problem in [17] for the finite case of nearly full rank with $\left(R_{0}, R_{1}, R_{2}\right)$ in $\mathbb{E}^{4}$; we shall merely quote those results where appropriate and not repeat the analysis. However, the other tools we have available mean that we place less emphasis on the mirror vector here.

In the subsequent discussion of blends, we need a somewhat weaker condition than faithfulness. Let $P$ be a realization of an abstract regular $n$-polytope $\mathcal{P}$ whose ambient space $\mathbb{A}$ is a sphere or Euclidean space according as $P$ is bounded or unbounded. (Note that regular apeirotopes can have bounded realizations.) If $\mathcal{G}(P)$ is the group of $P$, with generators $\left(R_{0}, \ldots, R_{n-1}\right)$, then we call $P$ untrivial if $R_{j} \neq I$ for each $j=$ $0, \ldots, n-1$. If $R_{k}=I$ for some $k$, then we say that $P$ is $k$-trivial. Recall from [14] or [24, Lemma 5A6] that, since we always confine our attention to the ambient space, if $R_{k}=I$, then $R_{j}=I$ for each $j>k$. Now exactly the same inductive argument based on vertex-figures as in [16] shows that $\operatorname{dim} R_{j} \geq j$, at least so long as $P$ is not $j$ trivial, except that we can have $\operatorname{dim} R_{n-1}=n-2$. Applying this to $k$-faces (bearing 
in mind that a realization may collapse onto a $k$-face, thus replacing $n$ by $k$ in the previous sentence), we deduce the triviality criterion:

Theorem 3.4 A realization $P$ of an abstract regular polytope $\mathcal{P}$ with ambient space $\mathbb{A}$ (a sphere or Euclidean space, as appropriate) is $k$-trivial for each $k>\operatorname{dim} \mathbb{A}$.

Remark 3.5 In the case of a finite (or bounded) realization $P$ in a Euclidean space $\mathbb{E}$, the degeneracy criterion of Theorem 3.4 says that $P$ is $k$-trivial for each $k \geq \operatorname{dim} \mathbb{E}$.

If we have (not necessarily faithful or even polytopal) realizations of the abstract regular polytope (or apeirotope) $\mathcal{P}$ in two Euclidean spaces, say $P$ with mirrors $S_{0}, \ldots, S_{n-1}$ in $\mathbb{X}$ and $Q$ with mirrors $T_{0}, \ldots, T_{n-1}$ in $\mathbb{Y}$ (possibly some $S_{j}=L$ or $T_{j}=M$ ), then their blend has mirrors $R_{j}:=S_{j} \times T_{j}$ in $\mathbb{E}:=\mathbb{X} \times \mathbb{Y}$ for $j=0, \ldots, n-1$. Indeed, if $v \in S_{1} \cap \cdots \cap S_{n-1}$ and $w \in T_{1} \cap \cdots \cap T_{n-1}$ are the initial vertices of the two realizations, then $(v, w)$ can be chosen as the initial vertex of the blend, which we write $P \# Q$. A realization which cannot be expressed as a blend in a non-trivial way is called pure.

Theorem 3.4 has the following consequence.

Theorem 3.6 Let $P$ be an untrivial realization of some abstract regular polytope or apeirotope. If $P$ is of full rank, then $P$ must be pure. If $P$ is of nearly full rank but is not pure, then one component of $P$ is one-dimensional (and the other is pure).

Proof We consider the case of bounded $P$; the proof in the other case is analogous. Let $\operatorname{dim} P=d$. If $P$ is blended with a component of dimension $r>0$, then this component must be $k$-trivial for $k \geq r$. Similarly, the other component must be $k$-trivial for $k \geq d-r$. Hence $P$ itself is $k$-trivial for $k \geq \max \{r, d-r\}$, and we immediately deduce the claims of the theorem.

Remark 3.7 In practice, we often regard components of a blend as (pre-)polytopes in their own right, particularly in the context of Theorem 3.6. In this context, we can actually allow $P$ and $Q$ to be realizations of different regular polytopes $\mathcal{P}$ and $\mathcal{Q}$, regarding them as realizations of a common regular polytope which covers both $\mathcal{P}$ and $\mathcal{Q}$.

There is a last restriction on realizations of apeirotopes which we wish to impose, that of discreteness. (In general, a discrete realization which is a blend can have nondiscrete components, but this will not happen here.) This means that $P$ has a discrete vertex-set vert $P=v \mathcal{G}$, and hence a finite vertex-figure; in the present context, this will also imply that $\mathcal{G}$ itself is crystallographic. The special group of $P$ is the subgroup $\mathcal{S}:=\left\langle S_{0}, \ldots, S_{n-1}\right\rangle$ of the orthogonal group, where (identified with its mirror) $S_{j}:=$ $R_{j}-R_{j}$ for each $j$; of course, $S_{j}$ is the translate of $R_{j}$ which contains $o$. (With the convention $v=o$, we have $S_{j}=R_{j}$ for $j \geq 1$.) Then $\mathcal{S}$ must be crystallographic and, in particular, must be finite and cannot have ordinary rotations (with axes of codimension 2) of periods other than 2, 3, 4 or 6 .

We end the section with what we call the nearest points criterion; this provides an easy way to eliminate many possibilities in Sect. 14. If $X$ is a finite set of points 
in an ambient space $\mathbb{A}$ (a sphere or Euclidean space) and $v \notin X$ is a further point, we denote by $\alpha(v, X)$ the smallest angle subtended at $v$ in $\mathbb{A}$ by some pair of points of $X$. Then the following should be obvious.

Proposition 3.8 Let $P$ be a regular polytope (apeirotope) with initial vertex $v$, and let $X$ be the set of vertices of $P$ (other than $v$ ) nearest to $v$. Then $\alpha(v, X)>\pi / 3$ $(\alpha(v, X) \geq \pi / 3$, respectively).

Proof If the given condition failed to hold, then two points of $X$ would be closer to each other than they are to $v$, a contradiction to the definition of $X$ and the regularity of $P$. (Note that the angle of a spherical equilateral triangle is greater than $\pi / 3$.)

Remark 3.9 Observe that the example $\{5,3\}$ as the vertex-figure of $\left\{\frac{5}{2}, 5,3\right\}$ shows that Proposition 3.8 need not hold when $X$ is, instead, the vertex-set of the vertexfigure of $P$ at $v$. (The notation for the classical regular polytopes follows [4].)

\section{Notation}

In this section, we briefly review the notation which we and others have introduced in earlier work. We first look at regular polygons in their own right, and then insofar as they contribute to the description of polytopes of higher rank. At the present stage, the term 'polytope' is generic.

A regular polygon is a blend of planar regular polygons $\{q\}$ for some rational $q>2$, a segment $\{2\}$ and the linear apeirogon $\{\infty\}$, not all of which need be present (rotations through irrational multiples of $\pi$ do not occur here, and so no special notation for them is necessary). The notation for such a polygon is $\{p\}$, where

$$
p=\frac{s}{t_{1}, \ldots, t_{k}}
$$

is a generalized fraction; here, $s, t_{1}, \ldots, t_{k}$ are non-negative integers such that $0 \leq$ $t_{1}<\cdots<t_{k} \leq \frac{1}{2} s$, with greatest common divisor $\left(s, t_{1}, \ldots, t_{k}\right)=1$. Then

$$
\{p\}=\left\{\frac{s}{t_{1}}\right\} \# \cdots \#\left\{\frac{s}{t_{k}}\right\},
$$

where each fraction is taken to be reduced to its lowest terms; here, $t_{1}=0$ corresponds to $\{\infty\}$, and $t_{k}=\frac{1}{2} s$ corresponds to $\{2\}$.

We shall need a little later the concept of the supplement $\left\{p^{\prime \prime}\right\}$ of $\{p\}$. If $q \geq 2$ is rational, define $q^{\prime \prime}$ by

$$
\frac{1}{q}+\frac{1}{q^{\prime \prime}}=\frac{1}{2}
$$

with the obvious definitions $2^{\prime \prime}=\infty$ and $\infty^{\prime \prime}=2$. If $\{p\}=\left\{q_{1}\right\} \# \ldots \#\left\{q_{k}\right\}$ with $\infty \geq q_{1}>\cdots>q_{k} \geq 2$, then we define

$$
\left\{p^{\prime \prime}\right\}:=\left\{q_{k}^{\prime \prime}\right\} \# \cdots \#\left\{q_{1}^{\prime \prime}\right\} .
$$


The reversal of order of the components is for consistency with the previous convention.

A geometric regular polygon is determined by the pair $\left(S_{0}, S_{1}\right)$ of generating reflexions of its symmetry group, and the choice of an initial vertex $v \in S_{1}$. With a regular $n$-polytope $P$ with group generators $\left(R_{0}, \ldots, R_{n-1}\right)$ are associated two particular regular polygons:

- its Petrie polygon, with $S_{0}:=R_{0} R_{2} \cdots$ and $S_{1}:=R_{1} R_{3} \cdots$ (products of all alternating terms);

- its deep hole, with $S_{0}:=R_{0}$ and $S_{1}:=R_{1} R_{2} \cdots R_{n-2} R_{n-1} R_{n-2} \cdots R_{2} R_{1}$.

In each case, the initial vertex of the polygon is always taken to be the initial vertex of $P$ itself.

Remark 4.1 Here and elsewhere these concepts have obvious abstract analogues, which the notation $\{\cdot\}$ will indicate.

A fine Schläfli symbol for a regular polytope $P$ takes the same form as its Schläfli type, except that now the entries are generalized fractions, and there may be additional specifications. Polyhedra (that is, the polytopes of rank 3 ) are the basic building blocks of polytopes of higher rank. Unfortunately, the general notation for polytopes based on polyhedra can get very cumbersome. As in the abstract case, a regular $n$ polytope whose (initial) facet is $P$ and (initial) vertex-figure is $Q$ is denoted $\{P, Q\}$, but this may only yield a partial description of it. (For instance, $\{\{3,3\},\{3,4\}\}$ is an alternative designation of the 4-cross-polytope $\beta_{4}=\{3,3,4\}$, although in practice one would never use it.) Note that the vertex-figure of $P$ must coincide with the facet of $Q$. If it is not actually universal, then extra relations are needed to determine its group. In two particular cases, we have some simple notation:

- with given Petrie polygon $\{r\}$, the notation is $\{P, Q: r\}$;

- with given deep hole $\{h\}$, the notation is $\{P, Q \mid h\}$.

The two notations can be combined to give $\{P, Q: r \mid h\}$ if necessary. As with the other entries in the Schläfli symbol, since we are attempting a geometric description of our polytope, it will usually be the case that $r$ and $h$ are generalized fractions. For an abstract description of the group, it is the numerators of the fractions in the whole notation which are significant (a 0 in the denominator means that we think of the corresponding number as $\infty$, but since this imposes no relation on the group, we do not mention it unless it is the first entry of the Schläfli type).

Remark 4.2 In many cases, we do not have a complete description of the group of a geometric regular polytope or apeirotope, and then a notation such as $\{P, Q: r\}$ or $\{P, Q \mid h\}$ (particularly with generalized fractional entries) is intended only to indicate the type of the Petrie polygon or deep hole. When we know that the notation actually specifies the group, we shall say so.

Remark 4.3 In contrast, there are many cases where a fine Schläfli symbol specifies the polytope geometrically, but not abstractly. For example, the great dodecahedron is 
geometrically (up to similarity) $\left\{5, \frac{5}{2}\right\}$, whereas abstractly it is $\{5,5 \mid 3\}$; the notation distinguishes it from the isomorphic small stellated dodecahedron $\left\{\frac{5}{2}, 5\right\}$. The notion of such specification is called rigidity; we shall discuss it in more detail in [19].

As we have said, we can deploy similar notation in the abstract case. However, one often needs to indicate less obvious group relations. Let $\boldsymbol{G}=\left\langle\boldsymbol{r}_{0}, \ldots, \boldsymbol{r}_{n-1}\right\rangle$ be the group of a regular polytope $\mathcal{P}$ of Schläfli type $\left\{p_{1}, \ldots, p_{n-1}\right\}$. If $\boldsymbol{r}_{j(1)} \cdots \boldsymbol{r}_{j(m)}=\boldsymbol{e}$ is a relation in $\boldsymbol{G}$, then we write $\mathrm{J}:=j(1) \ldots j(m)$ for the corresponding relator (thus, using the indices alone detaches the relator from the particular notation employed for the generators), so with $\boldsymbol{r}_{j}$ is associated the index $j$. If $\boldsymbol{G}$ is determined by the Schläfli type and the relators $J_{1}, \ldots, J_{r}$ (excluding those giving the Schläfli type), then we write

$$
\mathcal{P}:=\left\{p_{1}, \ldots, p_{n-1}\right\} /\left\langle\left\langle\mathrm{J}_{1}, \ldots, \mathrm{J}_{r}\right\rangle\right\rangle .
$$

For instance, with all entries integral,

$$
\begin{aligned}
\left\{p_{1}, \ldots, p_{n-1}: r\right\} & \left.=\left\{p_{1}, \ldots, p_{n-1}\right\} / \|(012 \ldots(n-1))^{r}\right\rangle, \\
\left\{p_{1}, \ldots, p_{n-1} \mid h\right\} & =\left\{p_{1}, \ldots, p_{n-1}\right\} / \|(012 \ldots(n-2)(n-1)(n-2) \ldots 21)^{h} \rrbracket .
\end{aligned}
$$

There is further notation to give a more precise description of polyhedra, but we will explain it in the appropriate place when we need it.

\section{Operations}

There are various operations which yield new regular polytopes from old ones. Two important techniques for obtaining groups of new regular polytopes are mixing and twisting. By mixing we mean picking out from an existing group (often, but not always, itself the group of a polytope) elements which themselves form a suitable canonical generating set. We shall later meet examples of this: Petrie contraction in Sect. 10, which will be extended in Sect. 11, as well as various things like the Petrie operation $\pi$. By twisting we mean adjoining to such a group one or more (usually involutory) automorphisms. The two techniques are not exclusive, as we shall see in Sect. 12, particularly since we find it convenient in our treatment to allow inner automorphisms as well (thus, strictly speaking, this is mixing rather than twisting).

In this section, with one exception we are concerned with operations which are reversible (in fact, they will be involutory). These have all been discussed in earlier papers already cited, and so our treatment of them will be fairly short.

So, let $P$ be a regular $n$-polytope (or apeirotope), whose group $\mathcal{G}=\mathcal{G}(P)$ has generating reflexions $\left(R_{0}, \ldots, R_{n-1}\right)$. It is important to remember that we regard $R_{j}$ indifferently as an involutory isometry or as its mirror (of fixed points). If $R$ is a linear reflexion, then so is $-R=(-I) R=R^{\perp}$, where the second expression $(-I) R$ thinks of it as an isometry, while the third $R^{\perp}$ identifies it with its mirror. Indeed, it may be appropriate to write $-I=\{o\}$, since it is the reflexion with mirror the origin; hence, the reflexion in a general point $w$ may be written $\{w\}$ on the same principle. 
If $P$ is finite, in which case we may suppose that its centre is the origin $o$, then we clearly obtain a new group if we replace $R_{j}$ by $-R_{j}$. If $P$ is an apeirotope, then we usually take its initial vertex to be the origin $o$, in which case we have the same possibility for $j \geq 1$. However, such sign changes will usually not give rise to a new polytope. Two cases where we often obtain polytopes are $j=0$ if $P$ is finite, or $j=1$ if $P$ is infinite. We discuss these first and then briefly mention further operations of a similar kind.

So, let $P$ be finite of rank $n$, with $\mathcal{G}=\mathcal{G}(P)$ generated by $\left(R_{0}, \ldots, R_{n-1}\right)$. To set things in a general context, define $Z:=R_{0} \cap \cdots \cap R_{n-1}$ (as a mirror). When $P$ has centre $o$ in its ambient space $E$, we have $Z=\{o\}$; for simplicity, we shall assume this. We then define $P^{\zeta}$ by its group $\mathcal{G}^{\zeta}$ with generators $\left(R_{0} Z, R_{1}, \ldots, R_{n-1}\right)=$ : $\left(S_{0}, \ldots, S_{n-1}\right)$; thus (in effect) $\zeta$ changes the sign of $R_{0}$ in $E$. More generally, replacing $R_{j}$ by $R_{j} Z$ is an operation denoted by $\zeta_{j}$, so that $\zeta=\zeta_{0}$; we rarely need the cases $j \geq 1$ (see also Remark 5.2).

Remark 5.1 If $P$ is a blend of the form $P=P^{\prime} \#\{2\}$, then it is easy to see that $P^{\zeta}=\left(P^{\prime}\right)^{\zeta}$, with the dimension lowered by 1 ; we shall tacitly exclude this case in the following discussion, but the reader should observe how it plays a rôle in the following Theorem 5.3.

Remark 5.2 The lower bound on $\operatorname{dim} R_{j}$ which we used for Theorem 3.4 also excludes the deployment of $\zeta_{j}$ in most cases.

The effect of $\zeta$ on the vertex-figure $Q$ of $P$ is straightforward: the vertex-figure of $P^{\zeta}$ is $-Q=Q Z$ (we could replace the initial vertex $v$ by $v Z$ to preserve $Q$, but it is more convenient not to). The effect of $\zeta$ on a proper (initial) face $F<P$ depends on two things: whether the centre $c=c(F)$ of $F$ coincides with $o$ or not, and whether $F$ is a blend with one component $\{2\}$ or not.

Theorem 5.3 Let $F$ be an initial (proper) face with centre $c$ of a finite regular polytope $P$ with centre $o$. Then, under $\zeta$, the corresponding initial face $\widehat{F}$ of $P^{\zeta}$ with centre $\widehat{c}$ is as follows:

(a) if $F$ is not a blend with component $\{2\}$ and $c=o$, then $\widehat{F}=F^{\zeta}$ with $\widehat{c}=o$;

(b) if $F$ is not a blend with component $\{2\}$ and $c \neq o$, then $\widehat{F}=F^{\zeta} \#\{2\}$ with $\widehat{c}=o$;

(c) if $F=G \#\{2\}$ is a blend with component $\{2\}$ and $c=o$, then $\widehat{F}=G^{\zeta}$ with $\widehat{c} \neq o$;

(d) if $F=G \#\{2\}$ is a blend with component $\{2\}$ and $c \neq o$, then $\widehat{F}=G^{\zeta} \#\{2\}$ with $\widehat{c} \neq o$ and $\langle c, \widehat{c}\rangle=0$.

Proof Except for the last claim that $\langle c, \widehat{c}\rangle=0$, for which see the picture following, this is all fairly obvious. In the picture, $G^{\prime}$ is a translate of the polytope $G$ involved in the blend, and edges of $F$ go from a vertex of $G$ to the corresponding adjacent vertex of $G^{\prime}$. As indicated, $G^{\prime \prime}=-G^{\prime}=G^{\prime} Z$, and edges of $\widehat{F}$ similarly go from a vertex of $G$ to the corresponding adjacent vertex of $G^{\prime \prime}$. Observe, by the way, that this illustrates the pairing $G \diamond\{2\}=G^{\zeta} \#\{2\}$ which we discuss in Sect. 6 . 


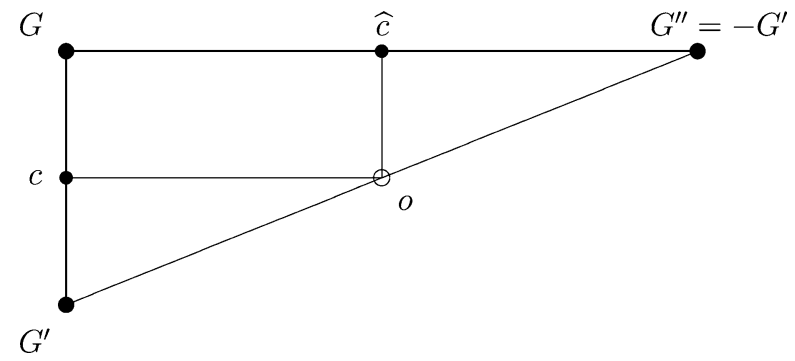

As we have seen, blends with segments behave somewhat anomalously under $\zeta$. Otherwise, we have the following.

Theorem 5.4 If $P$ and $Q$ are finite regular polytopes, neither of which is a blend with one component a segment, then $(P \# Q)^{\zeta}=P^{\zeta} \# Q^{\zeta}$. In particular, if $\{p\}$ is a regular polygon with no component $\{2\}$ or $\{\infty\}$, then $\{p\}^{\zeta}=\left\{p^{\prime \prime}\right\}$.

Proof Only the latter claim needs to be checked, and for this it is clear that, if $\{q\}$ is a (finite) planar regular polygon, then $\{q\}^{\zeta}=\left\{q^{\prime \prime}\right\}$. Recall that $q^{\prime \prime}$ is defined by $1 / q+1 / q^{\prime \prime}=1 / 2$.

An operation closely related to $\zeta$ is defined as follows; it applies to apeirotopes as well as polytopes. We now write $K_{j}:=R_{j} \cap \cdots \cap R_{n-1}$ (as the mirror of an involution). Then we have

Lemma 5.5 The involution $K_{j}$ commutes with all $R_{i}$ except $R_{j-1}$.

Proof To see this, we may suppose that $j \geq 1$ and note that $K_{j}$ is the Wythoff space of the $(n-j+1)$-coface $Q$ (say) of $P$. Since $R_{j-1}$ moves an initial vertex of $Q$ in $K_{j}$, it cannot commute with $K_{j}$. On the other hand, if $F$ is the initial $(j-1)$-face of $P$ and $k \geq j$, then it is not hard to see that $R_{k}$ fixes each $x \in \operatorname{vert} F$, so that $x \in R_{k}$. Hence vert $F \subseteq K_{j}$, and it follows that $K_{j}$ must commute with each $R_{i}$ for $i \leq j-2$. Finally, if $i \geq j$, then $K_{j} \subseteq R_{i}$, and so clearly $K_{j}$ commutes with $R_{i}$.

We let $\kappa_{i j}$ denote the operation of replacing $R_{i}$ by $R_{i} K_{j}$. Observe that $R_{i} K_{j}$ will be an involution for $i \neq j-1$ but will not commute with $R_{j-1}$; thus the only allowable cases are $i=j-2$ or $i=j$. We shall rarely need the former case, and so we usually write $\kappa_{j}:=\kappa_{j j}$ in the latter. Indeed, when $P$ is a regular apeirotope, we further write $\kappa:=\kappa_{1}$. If we suppose that $o$ is the centre of its vertex-figure $Q$, and so usually the initial vertex of $P$ itself, we see that $\kappa$ is the operation $\zeta$ applied to $Q$. We then let $P^{\kappa}$ be the corresponding new apeirotope, if it exists. In view of Remark 5.1, we initially assume that $Q$ is not a blend of the form $Q=Q^{\prime} \#\{2\}$; we shall see what happens if it is in Sect. 9.

Some properties of $\kappa$ follow easily from the definition, but for others, it is better to wait until Sect. 9.

Theorem 5.6 Under the operation $\kappa$ : 
(a) $(P \# Q)^{\kappa}=P^{\kappa} \# Q^{\kappa}$ for any regular apeirotopes $P$ and $Q$;

(b) if the initial 2-face of $P$ is $\{p\}$, then that of $P^{\kappa}$ is $\left\{p^{\prime \prime}\right\}$.

There are many operations which apply to polyhedra (or apeirohedra), but nearly all of them have no natural generalizations to the polytopes considered here. There are two exceptions. The first is the Petrie operation $\pi$, given by

$$
\left(R_{0}, \ldots, R_{n-1}\right) \mapsto\left(R_{0}, \ldots, R_{n-4}, R_{n-3} R_{n-1}, R_{n-2}, R_{n-1}\right)=:\left(S_{0}, \ldots, S_{n-1}\right) ;
$$

observe that $\pi=\kappa_{n-3, n-1}$. However, in certain circumstances, $\pi$ is an invalid operation. The first case here has often been employed by us in the past; the other two (which are new) fail for much the same reason.

Theorem 5.7 Let $P$ be a regular $n$-polytope of Schläfli type $\left\{p_{1}, \ldots, p_{n-1}\right\}$. Then the Petrie operation $\pi$ fails to yield a regular polytope $P^{\pi}$ in at least the following cases:

(a) if $n \geq 4$ and $q:=p_{n-3}$ is odd;

(b) if $n \geq 5$ and the size $q$ of the hole of the $(n-5, n-3)$-section of $P$ (of type $\left.\left\{p_{n-4}, p_{n-3}\right\}\right)$ is odd;

(c) if $n \geq 5$ and the size $q$ of the Petrie polygon of the $(n-5, n-3)$-section of $P$ is odd.

Proof Note that these results actually hold on an abstract level. For (a), in the notation above for the operation we have

$$
S_{n-1}=R_{n-1}=\left(R_{n-4} R_{n-3} \cdot R_{n-1}\right)^{q}=\left(S_{n-4} S_{n-3}\right)^{q} \in\left\langle S_{n-4}, S_{n-3}\right\rangle,
$$

violating the intersection property. For (b), we similarly have

$$
\begin{aligned}
S_{n-1}=R_{n-1} & =\left(R_{n-5} R_{n-4} R_{n-3} R_{n-4} \cdot R_{n-1}\right)^{q} \\
& =\left(S_{n-5} S_{n-4} S_{n-3} S_{n-4}\right)^{q} \in\left\langle S_{n-5}, S_{n-4}, S_{n-3}\right\rangle,
\end{aligned}
$$

and for (c), we have

$S_{n-1}=R_{n-1}=\left(R_{n-5} R_{n-4} R_{n-3} \cdot R_{n-1}\right)^{q}=\left(S_{n-5} S_{n-4} S_{n-3}\right)^{q} \in\left\langle S_{n-5}, S_{n-4}, S_{n-3}\right\rangle$,

both violating the intersection property.

The second such operation is what we shall call halving, though the name is even less appropriate than in the case of rank 3 where it originates. This applies to a polytope $P$ of Schläfli type $\left\{p_{1}, \ldots, p_{n-1}\right\}$ with $p_{n-2}=4$ and is

$$
\begin{aligned}
\eta: & \left(R_{0}, \ldots, R_{n-1}\right) \mapsto\left(R_{0}, \ldots, R_{n-4}, R_{n-3} R_{n-2} R_{n-3}, R_{n-1}, R_{n-2}\right) \\
\quad= & :\left(S_{0}, \ldots, S_{n-1}\right) .
\end{aligned}
$$

If a genuine polytope $Q$ is obtained, then the Schläfli type of $Q$ is, say, $\left\{q_{1}, \ldots, q_{n-1}\right\}$, where $q_{j}=p_{j}$ for $j \neq n-2$, while $q_{n-2}$ is the type of the hole of the original 
$(n-4, n-1)$-section of type $\left\{p_{n-3}, p_{n-2}\right\}$. The new group is a proper subgroup of the old one, except when the edge-graph of the $(n-4, n-1)$-section has odd circuits.

It is worth pointing out that $\eta$ has been applied informally in earlier work to polytopes of higher rank than 3, for instance, [18], as well as in [24, Sect. 10E] (with a different notation-we shall meet the example in Sect. 12).

\section{Blends}

If a finite regular polytope $P$ of nearly full rank is a blend, then Theorem 3.6 implies that one of its components must be a segment $\{2\}$; the other component is then a regular polytope $Q$ of full rank. The situation for a regular apeirotope $P$ of nearly full rank is very similar; one component is a segment $\{2\}$ or apeirogon $\{\infty\}$, while the other is a regular apeirotope $Q$ of full rank. Actually, at this stage what we are claiming is not fully established, because in theory it is possible for the (necessarily untrivial) pure component $Q$ of full rank not to be polytopal. Thus one purpose of the section is to prove the relevant Theorem 6.4 and 6.5 below. We consider the two cases in turn.

Remark 6.1 The blend of a finite regular polytope of full rank $n$ and an apeirogon is an apeirotope of rank $n$ and dimension $n+1$, and is therefore not of nearly full rank.

We have already met one important feature of blends $P=Q \#\{2\}$ in the fourdimensional case (in fact, it even applies to blends with $Q$ a polygon). We have seen in Sect. 5 that finite regular polytopes are paired by the operation $\zeta$, which replaces the initial generating reflexion $S_{0}$ of (say) $Q$ (with centre at $o$ ) by $-S_{0}=S_{0}^{\perp}$ to give $Q^{\zeta}$, but we observed in Remark 5.1 that $\zeta$, when applied to blends with segments, lowers the dimension. We can retrieve this situation by forming a blend in an alternative way. If $Q$ has codimension 1, then translate $Q$ in a direction orthogonal to $\operatorname{lin} Q$ and (in effect) apply the same operation $\zeta$; the resulting polytope is denoted $Q \diamond\{2\}$. Then blends are also paired by

$$
Q \diamond\{2\}=Q^{\zeta} \#\{2\} .
$$

To be more specific, suppose that $Q$ (in $\mathbb{E}^{d}$ ) has group generators $\left(S_{0}, \ldots, S_{d-1}\right)$. In $\mathbb{E}^{d+1}=\mathbb{E}^{d} \times \mathbb{R}$, the respective generators (regarded as mirrors) are

$$
\begin{array}{cl}
\left(S_{0}, S_{1} \times \mathbb{R}, \ldots, S_{d-1} \times \mathbb{R}\right) & \text { for } Q \#\{2\}, \\
\left(S_{0}^{\perp}, S_{1} \times \mathbb{R}, \ldots, S_{d-1} \times \mathbb{R}\right) & \text { for } Q \diamond\{2\} ;
\end{array}
$$

the orthogonal complement in the second expression is taken in $\mathbb{E}^{d}$, of course. We shall usually find it more convenient to write $Q \diamond\{2\}$ rather than $Q^{\zeta} \#\{2\}$.

Remark 6.2 In fact, the two polytopes $Q \#\{2\}$ and $Q \diamond\{2\}$ are isomorphic. With $Q$ a $d$-polytope as before, we can take their vertices (in $\mathbb{E}^{d+1}$, say) in the hyperplanes $\xi_{d+1}=\varepsilon= \pm 1$ in $\mathbb{E}^{d+1}=\mathbb{E}^{d} \times \mathbb{R}$. With $x \in \mathbb{E}^{d}$, the isomorphism is given by

$$
(x, \varepsilon) \mapsto(\varepsilon x, \varepsilon)
$$

for each $x \in \operatorname{vert} Q$. 
Remark 6.3 In the case of the $d$-simplex $\alpha_{d}$, we have the (combinatorial) isomorphism

$$
\alpha_{d} \#\{2\} \cong \alpha_{d} \diamond\{2\} \cong \alpha_{d}^{\zeta},
$$

because the last polytope (with twice as many vertices as $\alpha_{d}$ ) has even edge-circuits. Similarly, for the cube $\gamma_{d}$, we have

$$
\gamma_{d} \#\{2\} \cong \gamma_{d}^{\zeta} \#\{2\} \cong \gamma_{d}
$$

and so on, in view of (6.1). Note that $\gamma_{d}^{\zeta}$ has the vertices of the half-cube $h \gamma_{d}$ when $d$ is odd.

There is a further point. If $Q$ is a classical regular 4-polytope of full rank 4, then the Petrie operation $\pi$ only works in one case:

$$
\{4,3,3\}^{\pi}=\left\{\left\{4, \frac{4}{1,2} \mid 4\right\},\left\{\frac{4}{1,2}, 3: 3\right\}\right\}
$$

it fails in all the others, because their 2-faces are odd. It might be thought that, once we blend with $\{2\}$, the Petrie operation will now be valid for these others, because the 2 -faces of such blends are even polygons. However, these examples still fail, because their degeneracy actually runs more deeply than the oddness of their faces.

It is appropriate to explain what is happening here, particularly since we passed over these matters rather lightly in [16, 17]. We deal with the polyhedra of [17] first. The relevant cases here are those with mirror vectors $(3,2,3)$ or $(1,2,3)$ (as defined in Sect. 3), the latter being related to the former by $\zeta$ (denoted $\kappa_{0}$ in earlier papers). These are derived by twisting diagrams $\mathcal{D}_{1}(p, q ; r)$ of the form

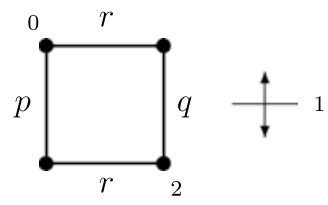

The labels $0,1,2$ on the diagram refer to the generators of the symmetry group of the corresponding regular polyhedron (if it exists); thus $R_{1}$ is a twist-an (inner or outer) automorphism of the diagram. In the excluded cases, $p$ is a fraction with an even denominator.

Now, if we apply $\zeta_{1}$, changing the sign of the second generator $R_{1}$ of the symmetry group, then we replace $p$ and $q$ by their complements $p^{\prime}$ and $q^{\prime}$, with

$$
\frac{1}{p}+\frac{1}{p^{\prime}}=1, \quad \frac{1}{q}+\frac{1}{q^{\prime}}=1 .
$$

For the previously excluded polyhedra, $p^{\prime}$ now has odd denominator (included here is the case when it is an integer). When $q^{\prime}$ is a fraction with even denominator, the discussion of these cases in [17] shows that they are obtained from classical regular 4polytopes by the Petrie contraction operator $\varpi$, which we shall consider in Sect. 10. Forward reference to that section then exhibits the excluded cases as applications of 
$\zeta \varpi$ to the 4-polytopes; the discussion following Proposition 10.3 shows that fewer vertices are obtained than the theoretical quotient of the order of the whole group by that of the vertex-figure. This is not surprising, because we know that vertices are identified by the degeneracy of the faces (at least, with mirror vector $(3,2,3)$ ). We then see that applying $\zeta$ cannot rescue this situation.

In the two remaining cases, with $(p, q, r)=\left(\frac{5}{3}, 2,5\right)$ or $\left(5, \frac{5}{2}, 2\right)$, to which the previous considerations do not apply, direct calculations show that the $1800=14400 / 8$ expected vertices coincide in threes with the 600 vertices of $\{5,3,3\}$. Thus applying $\zeta$ will not produce a genuine polyhedron in either case.

For the 4-polytopes of [16] (with the exception of $\{4,3,3\}$ ), if we apply $\pi$, then we obtain facets which are polyhedra of the type we have just excluded; in fact, we are applying the operation (7.2) of [17] in the dual situation (see also [21]). Thus-even apart from the degeneracy of the faces - the new facets degenerate, again in a way that applying $\zeta$ will not remedy.

Returning to the main discussion, we conclude that no unexpected examples of polytopal blends with segments arise in five-dimensional space. In yet higher dimensions, even theoretically there is no way of constructing examples other than the obvious ones. We can summarize the analysis so far (which confirms what we claimed in the first paragraph) in

Theorem 6.4 There are 34 blended finite regular polytopes of nearly full rank in $\mathbb{E}^{5}$, and 6 in $\mathbb{E}^{d}$ for each $d \geq 6$. Each is constructible in two ways, related by (6.1).

The count in Theorem 6.4 comes from the table of [16, p. 34], as does that in the following Theorem 6.5 .

The case of blended regular apeirotopes of nearly full rank can now be dealt with quickly. Exactly the same considerations as in the finite case prevent applications of the Petrie operation $\pi$, except where we already know that it is permitted for the pure component of full rank. That is, as with blending with $\{2\}$, blending with $\{\infty\}$ cannot undo degeneracies which arise from (mis)application of other operations. We thus arrive at the following:

Theorem 6.5 There are 36 blended regular apeirotopes of nearly full rank in $\mathbb{E}^{5}$, and 16 in $\mathbb{E}^{d}$ for each $d \geq 6$.

\section{Vertex-figures}

The vertex-figure $Q$ of a regular $n$-polytope $P$ of nearly full rank is a (finite) regular $(n-1)$-polytope of full or nearly full rank; at this stage, we treat the finite and infinite cases together. (If $P$ is finite with centre $o$, then the vertices of $Q$-thought of as the other vertices of the edges of $P$ through its initial vertex $v$-lie on the intersection of a sphere with centre $o$ and one with centre $v$, and so on a hyperplane which does not contain $v$.) Since it can be assumed that we have a list of regular $(n-1)$-polytopes of nearly full rank to hand, it is the case of full rank which we need to discuss further.

Let $P$ be a regular polytope or apeirotope of nearly full rank. It is helpful to distinguish three classes of vertex-figure $Q$ of $P$ : 
- $Q$ is of full rank;

- $Q$ is blended;

- $Q$ is pure.

We settle the first class in Theorem 7.1 immediately below, and the second in Sect. 10 (but see also the next Sect. 8). The third case ultimately relies on the first two, as working back through successive vertex-figures easily shows; the key classification is that of the finite regular polyhedra of nearly full rank, which was accomplished in [17].

The basic result here is the following.

Theorem 7.1 If $n \geq 4$, and $P$ is an n-polytope of nearly full rank whose vertex-figure $Q$ is an $(n-1)$-polytope of full rank, then $P$ is a blend.

Proof The proof is just about the same as that of [18, Theorem 6.2] and, indeed, is the same if we adopt the viewpoint of [16] and earlier discussions in this paper, and regard the ambient space of a finite regular polytope as the sphere containing its vertices.

So, suppose that $P$ is a regular $n$-polytope in the $n$-dimensional ambient space $E$ (spherical or Euclidean as appropriate), whose vertex-figure $Q$ is $(n-1)$-dimensional in $E$, so that $Q$ is of full rank. As usual, the group of $P$ is $\mathcal{G}(P)=\left\langle R_{0}, \ldots, R_{n-1}\right\rangle$, with the $R_{j}$ the canonical generating reflexions; we take $v$ to be the initial vertex of $P$ and $w$ that of $Q$ (we emphasize that, in the present context, vert $Q$ consists of the mid-points of the edges of $P$ through $v$, so that $w$ is the mid-point of the initial edge). Let $H:=\operatorname{span} Q(=\operatorname{span} \operatorname{vert} Q)$, so that $H$ is a hyperplane. Let $J$ be the hyperplane through $w$ perpendicular to the line $M:=\operatorname{span}\{v, w\}$; since reflexion in $R_{0}$ interchanges $v$ and the other vertex of $P$ on the initial edge, it is clear that $w \in R_{0} \subseteq J$.

Further, write $K:=H \cap J, L$ for the line in $J$ through $w$ perpendicular to $K$, and $S_{j}:=H \cap R_{j}$ for $j=1, \ldots, n-1$. Now $w$ is the sole point in $H$ fixed by $S_{1}, \ldots, S_{n-1}$; we infer that the only possibilities for $R_{0}$ are $R_{0}=\{w\}, L, K$ or $J$.

In fact, there are two possibilities. If $v$ coincides with the centre $c$ of $Q$, then all vertices of $P$ lie in $H$, so that $P$ is $(n-1)$-dimensional (and so of full rank), contrary to the initial supposition. Thus, $v \neq c$ must lie on the line $N$ through $c$ perpendicular to $H$. In this case, it follows that $R_{j}=\operatorname{span}\left(N \cup S_{j}\right)$ for each $j \geq 1$. Moreover, since $L$ now meets $N$ (in $b$, say), we see that vert $P$ lies in a sphere with centre $b$. Therefore, again, if $R_{0}=L$ or $J$, then $P$ turns out to be of full rank (and finite in case $E$ is Euclidean). We conclude that $R_{0}=\{w\}$ or $K$, and it follows immediately that $P$ is actually a blend.

Remark 7.2 It is worth looking into why this result fails for $n=3$. In fact, where it will go wrong is when $R_{2}$ is the half-turn about the initial edge; then $R_{0}$ can be any line in $J$ through $w$, and with no further restriction the argument does not carry over.

In [18, Sect. 6], we went on to discuss blended vertex-figures. We could do the same thing here. However, it is preferable to postpone this discussion until later sections; the situation is somewhat more restrictive than in [18]. 


\section{The Gateway}

It is unnecessary to point out that the symmetry group $\mathcal{H}=\mathcal{G}(Q)$ of the vertex-figure $Q$ of a regular polytope (or apeirotope) $P$ is a subgroup of the group $\mathcal{G}=\mathcal{G}(P)$ of $P$, whose index $[\mathcal{G}: \mathcal{H}]$ is the number of vertices of $P$. However, for a polytope of nearly full rank in $\mathbb{E}^{d}$ with $d \geq 5$ which is not a blend (as we may assume henceforth), $\mathcal{G}$ will be a subgroup of a hyperplane reflexion group, possibly with outer automorphisms; this will even hold for polytopes whose groups consist of direct isometries only, as we shall see in Sect. 14. This severely restricts what $\mathcal{H}$ can be. Moreover, because $\mathcal{H}$ will (in effect) be a subgroup of $\mathcal{G}$ in only one way, it will immediately determine the vertex-set of the polytope $P$; in turn, since vert $Q \subseteq$ vert $P$ (in the obvious way), this places further restrictions on $Q$.

This is particularly important for the finite polytopes in the 'gateway' dimension $d=5$, because here $\mathcal{G}$ must be a subgroup of $[3,3,3,3] \rtimes C_{2}=[3,3,3,3] \times C_{2}$ or $[3,3,3,4]$; we have already classified the regular polytopes and apeirotopes of full or nearly full rank in lower dimensions. In this section, therefore, we treat these 'gateway' 4-polytopes in general terms, except that we leave the cases of those with direct symmetries only to Sect. 14 .

Remark 8.1 The term 'gateway' comes from genealogy. A 'gateway' ancestor is one through whom many lines of descent are traced (from notable ancestors to notable descendants); in English history, Edward III is often cited as an instance.

So, let us make the procedure explicit:

- for given $\mathcal{H}$, identify those groups $\mathcal{G}$ for which $\mathcal{H} \leq \mathcal{G}$;

- from the fixed point of $\mathcal{H}$ in $\mathcal{G}$, identify the vertex-set $V$ of $P$;

- check whether the vertex-set of $Q$ is an appropriate subset of $V$.

We deal with blended vertex-figures first. Noting that the blends $\gamma_{3} \#\{2\}$ and $\gamma_{3} \diamond$ $\{2\}$ with a 3 -cube $\gamma_{3}$ actually have group $[3,3] \times \mathcal{C}_{2}$, we see that this and $[3,4] \times \mathcal{C}_{2}$ are the only possible groups $\mathcal{H}$. In effect, we can recover the symmetry giving the blend. In the case of cubes, we pass to the Petrial first (and then return afterwards). If the vertex-figure $Q$ has group $\mathcal{H}=\mathcal{H}(Q)=\left\langle R_{1}, R_{2}, R_{3}\right\rangle$, then $\left(R_{1} R_{2}\right)^{6}=I$, and $T:=$ $\left(R_{1} R_{2}\right)^{3}$ is the blending element. Although, strictly speaking, they are isomorphic groups, we find it convenient to distinguish [3,3] $\times \mathcal{C}_{2}$ and $[3,3] \rtimes \mathcal{C}_{2}$. In the latter case, we can have $\mathcal{G}=[3,3,3,3] \rtimes \mathcal{C}_{2}$, with vertex-set that of $D \alpha_{5}$ (the difference body), whose vertices are those of the (Minkowski) sum of $\alpha_{5}$ and its reflexion $-\alpha_{5}$ in its centre. For the remaining cases, the vertices are the mid-points of the edges of a regular 5-polytope; see Sect. 10.

For other than blends, we must therefore look at potential vertex-figures $Q$ with mirror vectors of types $(r, 2,3)$ for $r=1,2,3$ or $(2,3,2)$. Now we must exclude groups which contain $[r] \times[r]$ with $r \geq 5,[3,4,3]$ or $[3,3,5]$ from the outset, so reference to [17] shows that the only groups which survive are subgroups of $([r] \times$ $[r]) \rtimes \mathcal{C}_{2}$ (with $r=3,4$ ), [3, 3, 3] $\rtimes \mathcal{C}_{2}$ and [3, 3, 4]. We consider these in turn.

The group $\mathcal{H}=([3] \times[3]) \rtimes \mathcal{C}_{2}$ is a subgroup of $\mathcal{G}=[3,3,3,3] \rtimes \mathcal{C}_{2}$, but not of $[3,3,3,4]$. Moreover, the corresponding polytope $P$ must have the vertices of the 
truncate $0_{22}$ of the 5-simplex $\alpha_{5}$; these are the centres of the triangular 2-faces of $\alpha_{5}$. Observe that the vertex-figure of $0_{22}$ is the product $\{3\} \times\{3\}$.

The group ([4] $\times[4]) \rtimes \mathcal{C}_{2}$ is a subgroup of [3,3,3,4], actually in two distinct ways. However, the putative polytope $P$ would then have to have the vertices of the 5 -cross-polytope $\beta_{5}$. This immediately excludes the torus $\left\{4, \frac{4}{1,2} \mid 4\right\}$ with 16 vertices as a possible vertex-figure. It might seem to permit $\left\{4, \frac{4}{1,2} \mid 4\right\}^{\eta}=\left\{\frac{4}{1,2}, 4: \frac{4}{1,2}\right\}$ with the 8 vertices of the half-cube $h \gamma_{4}$, which are the same as those of $\beta_{4}$. However, the induced symmetry $R_{1}$ fixes the initial vertex in $\beta_{5}$, which cannot be allowed; hence this case does not occur.

The group $\mathcal{H}=[3,3,3]$ is a subgroup of both [3, 3, 3, 3] and $\mathcal{G}=[3,3,3$, 4]. However, in the former case, a putative polytope $P$ would have to have the vertices of $\alpha_{5}$, possibly together with the copy $-\alpha_{5}$ of $\alpha_{5}$ obtained by reflexion in its centre; the vertex-figure would then have to have the vertices of $\alpha_{4}$, which is not possible. There remains the latter case. The vertices of $P$ must then be a subset of those of the 5-cube $\gamma_{5}$, so that we should write $\mathcal{G}=[4,3,3,3]$; consequently, the vertex-figure must then have the vertices of the truncate $0_{21}$ of $\alpha_{4}$ (again, $\alpha_{4}$ itself cannot occur), which are the mid-points of the edges of $\alpha_{4}$. Also covered here is the case of the half-cube $h \gamma_{5}$, with $\mathcal{G}=\left[3^{2,1,1}\right]$.

Finally, though $[3,3,3] \rtimes \mathcal{C}_{2}$ is a subgroup of $[3,3,3,3] \rtimes \mathcal{C}_{2}$, we come back to the first case in the previous paragraph, which we have already excluded.

Remark 8.2 Of course, the same kind of analysis applies in higher rank, but now we will have an even more severely restricted list of (vertex-sets of) potential vertexfigures.

\section{Apeirotope Constructions}

In this section we discuss two constructions which lead from finite regular polytopes to regular apeirotopes, with one preserving rank deficit and the other increasing rank deficit by 1 . A connexion between them is shown in Sect. 10, using a third construction. All these constructions have appeared before (in special cases), but the relationship has not previously been recognized.

The first construction is that of the free abelian apeirotope. If $Q$ is a (finite) rational regular polytope, meaning that its vertices all have rational coordinates with respect to some coordinate system, and $L$ is the subspace of points fixed by $\mathcal{G}(Q)$ whose generating set is $\left(S_{0}, S_{1}, \ldots\right)$ (thus $L \leq S_{k}$ for each $k$ ), then $P:=$ apeir $Q$ has a point $v \in L$ as initial vertex and generating set for $\mathcal{G}(P)$ given by $R_{0}:=\{w\}$ where $w$ is the initial vertex of $Q$, and $R_{j}:=S_{j-1}$ for $j \geq 1$.

Though the construction appears to have a lot of latitude, in fact the only difference is whether $v \in$ aff $Q$ or not. If $P$ is the apeirotope obtained when $v \in$ aff $Q$, then the other case is $P \#\{2\}$. In general, the two cases are isomorphic; they are not just when $P$ has odd edge-circuits, which (in practice) only happens in a few cases where $Q$ has a subset of vertices forming a diametral (planar regular) hexagon.

Remark 9.1 For the apeir construction to fail to yield a regular apeirotope, the following are necessary: 
- $v \in$ aff $Q$;

- apeir $Q$ has odd edge-circuits;

- $Q$ is not centrally symmetric or is handed (has a purely rotational symmetry group—see Sect. 14).

We note that such examples occurred in [18].

In five or more dimensions, we can take for granted the fact that-except for the blends involving the pentagonal regular polytopes of full rank in $\mathbb{E}^{4}$-all regular polytopes $Q$ under discussion are rational and hence permit apeirotopes apeir $Q$. Only when such apeirotopes are related in a natural way to others, will they receive specific mention. For the construction to yield an apeirotope apeir $Q$ of nearly full rank, we thus need one of the following:

- $Q$ of nearly full rank and initial vertex $v \in$ aff $Q$,

- $Q$ of full rank and $v \notin$ aff $Q$.

Remark 9.2 We should note that, with $Q$ of full rank, apeir $Q \#\{2\} \cong$ apeir $Q$ in nearly all cases (eight in $\mathbb{E}^{5}$ and six in $\mathbb{E}^{d}$ for each $d \geq 6$ ). The exceptions are $Q=$ $\{3,4,3\}$ or $\{3,4,3\}^{\zeta}$, with diametral hexagons of $\{3,4,3\}$ forcing apeir $Q$ to have triangular edge-circuits.

For our other construction, we observe that what we have used as an operation in Sect. 5 actually yields a construction if suitably generalized. Let $Q$ be a finite regular polytope whose group $\mathcal{G}(Q)$ is crystallographic, let $\left(S_{0}, S_{1}, S_{2}, \ldots\right)$ be the generating set of $\mathcal{G}(Q)$, and let $w$ be the initial vertex of $Q$. The point-reflexion in $w$ is then $\{w\}$ (regarded as a mirror-we mentioned this in Sect. 5); the construction is then given by the new generating set

$$
\left(S_{0}, S_{1}, S_{2}, \ldots\right) \longmapsto\left(S_{0},\{w\} S_{1}, S_{2}, \ldots\right)=:\left(R_{0}, R_{1}, R_{2}, \ldots\right) .
$$

If $Q$ were an apeirotope, then we would have designated the corresponding operation by $\kappa$ (and recall that we confined its usage to apeirotopes). In keeping with what we did in [18], we use the same symbol $\kappa$ for this construction. We therefore have

Theorem 9.3 If $Q$ is a finite crystallographic regular polytope of full rank, then $Q^{\kappa}$ is a putative discrete regular apeirotope of nearly full rank. Conversely, if $P$ is a regular apeirotope of nearly full rank whose vertex-figure is blended, then $P^{\kappa}$ is a putative regular polytope of full rank.

Remark 9.4 We say "putative" here, because in some cases the construction fails to yield an apeirotope or polytope (whose group satisfies the intersection property). We shall see examples when we come to consider individual cases; there is one in Sect. 12.

Remark 9.5 When the construction is applied to a regular $d$-polytope with $d \leq 4$, it may be possible further to apply the Petrie operation $\pi$. When $d \geq 5$, however, this can no longer happen; in no case is $\pi$ applicable. 
The description of the faces of $P=Q^{\kappa}$ in terms of those of $Q$ exactly follows Sect. 5. However, it is worth our while to summarize the overall picture from the view-point of the classical regular polytopes in the sense of Coxeter [4], whose symmetry groups are generated by hyperplane reflexions.

Theorem 9.6 If $\{P, Q\}$ is a finite classical crystallographic regular polytope with facet $P$ and vertex-figure $Q$, then

(a) $\{P, Q\}^{\kappa}=\left\{P^{\kappa}, Q^{\zeta} \#\{2\}\right\}$,

(b) $\{P, Q\}^{\zeta \kappa}=\left\{P^{\zeta \kappa} \#\{\infty\}, Q^{\zeta} \#\{2\}\right\}$.

Proof All we need do is notice that $\{P, Q\}^{\zeta}=\left\{P^{\zeta} \#\{2\}, Q\right\}$; everything else follows from the previous discussion.

We therefore go through the individual cases, bearing in mind that, as shown in [16], in $\mathbb{E}^{d}$ (with $d \geq 5$ ) the only (finite) regular polytopes of full rank are the simplex, cross-polytope and cube, and the results of applying $\zeta$ to them. In view of Theorem 9.6, we need only discuss in detail the initial case of interest, which will be that of rank 3 .

In fact, we have the following.

$$
\begin{aligned}
&\{3,3\}^{\kappa}=\left\{6, \frac{6}{1,3} \mid 3\right\} \cong\{6,6 \mid 3\}, \\
&\{3,4\}^{\kappa}=\left\{6, \frac{4}{1,2} \mid 4\right\} \cong\{6,4 \mid 4\}, \\
&\{4,3\}^{\kappa}=\left\{4, \frac{6}{1,3} \mid 4\right\} \cong\{4,6 \mid 4\}, \\
&\{3,3\}^{\zeta \kappa}=\left\{\frac{3}{0,1}, \frac{6}{1,3}: \frac{4}{1,2}, \frac{4}{1,2}\right\} \cong\{\infty, 6: 4,4\}, \\
&\{3,4\}^{\zeta \kappa}=\left\{\frac{3}{0,1}, \frac{4}{1,2}: \frac{6}{1,3}, \frac{4}{1,2}\right\} \cong\{\infty, 4: 6,4\}, \\
&\{4,3\}^{\zeta \kappa}=\left\{\frac{4}{0,1}, \frac{6}{1,3}: \frac{6}{1,3}, 3\right\} \cong\{\infty, 6: 6,3\} .
\end{aligned}
$$

(Recall our use of heavy braces to denote abstract polytopes.) Of course, $\{3,4\}$ and $\{3,4\}^{\zeta}$ will not play any part as faces in $\mathbb{E}^{d}$ for $d \geq 5$. It is also worth noting that $\left\{3^{d-2}, 4\right\}^{\zeta}$ has the same vertices and edges as the original cross-polytope $\left\{3^{d-2}, 4\right\}$ and that

$$
\left\{4,3^{d-2}\right\}^{\zeta}= \begin{cases}\left\{\frac{4}{1,2}, 3^{d-2}\right\} \cong\left\{4,3^{d-2}\right\} & \text { if } d \text { is even } \\ \left\{\frac{4}{1,2}, 3^{d-2}: \frac{d}{1,2, \ldots, m}\right\} \cong\left\{4,3^{d-2}\right\} / 2 & \text { if } d \text { is odd }\end{cases}
$$

here, $m:=(d-1) / 2$, and $\left\{4,3^{d-2}\right\} / 2 \cong\left\{4,3^{d-2}: d\right\}$ is obtained by identifying opposite vertices of the original $d$-cube $\left\{4,3^{d-2}\right\}$. 


\section{Petrie Contraction}

We now come to a further construction, which generalizes one introduced in [20] (see also [17]). This uses the mixing operation

$$
\varpi:\left(S_{0}, \ldots, S_{n}\right) \mapsto\left(S_{1}, S_{0} S_{2}, S_{3}, \ldots, S_{n}\right)=:\left(R_{0}, \ldots, R_{n-1}\right),
$$

which (when it is valid) yields a regular $n$-polytope $P:=Q^{\varpi}$ of nearly full rank from a regular $(n+1)$-polytope $Q$ of full rank. (Of course, we use 'polytope' generically here; if $Q$ is an apeirotope, then $Q^{\varpi}$ is also.) We shall call $P$ the Petrie contraction of $Q$.

When $Q$ is a classical regular polytope, part of the description of $Q^{\varpi}$ in terms of $Q$ can be gleaned from the earlier papers $[18,20]$ :

- the vertices of $Q^{\varpi}$ are the mid-points of the edges of $Q$,

- the 2-faces of $Q^{\varpi}$ are central sections of the 3-faces of $Q$, whose edges join midpoints of successive edges of their Petrie polygons,

- the vertex-figure of $Q^{\varpi}$ is the blend $G \#\{2\}$, where $G$ is the edge-figure of $Q$.

If the Schläfli type of $Q$ is $\left\{q_{1}, \ldots, q_{n}\right\}$ with $q_{3}$ even, then $Q^{\varpi}$ splits. However, since we are working in $\mathbb{E}^{d}$ with $d \geq 5$, we do not encounter such examples. With $d \geq 5$, the only finite examples of $Q$ are the simplex, cube, cross-polytope and the results of applying $\zeta$ to them, and (apart from the six free abelian apeirotopes, which we treat next) the only apeirotopes are the cubic tiling and its transform under $\kappa$.

Remark 10.1 For $k \geq 3$, the $k$-face of $Q^{\varpi}$ is $F^{\varpi}$, where $F$ is the $(k+1)$-face of $Q$. Note also that, when $k=3$, the Petrie polygon of $F^{\varpi}$ is of the same kind as that of $F$, while the hole of $F^{\varpi}$ is that of the vertex-figure of $F$.

Similar arguments to those of Sect. 7 show that Petrie contraction can be reversed for a polytope whose vertex-figure is a blend with a simplex or cross-polytope; naturally, this does not apply to a blend with a cube.

The connexion already mentioned in Sect. 9 is the following.

Theorem 10.2 If $Q$ is a finite rational regular polytope of full rank, then $(\text { apeir } Q)^{\varpi}=Q^{\kappa}$ is a regular apeirotope of nearly full rank.

Proof We use the notation in the definition of apeir $Q$. Bearing in mind that $w$ is the initial vertex of $Q$ (and so of $Q^{\kappa}$ also), the generating set of the group of (apeir $\left.Q\right)^{\varpi}$ is

$$
\left(R_{0}, R_{1}, R_{2}, R_{3}, \ldots\right)=\left(\{w\}, S_{0}, S_{1}, S_{2}, \ldots\right) \stackrel{\varpi}{\longmapsto}\left(S_{0},\{w\} S_{1}, S_{2}, \ldots\right),
$$

which replaces $S_{1}$ by its image under the point-reflexion in $w \in S_{1}$. This just defines $Q^{\kappa}$, as claimed.

In general terms, we also have the following. 
Proposition 10.3 Let $Q$ be a finite regular $d$-polytope with $d \geq 5$. Then $Q^{\zeta \varpi}=$ $Q^{\varpi \zeta_{1}}$, if the polytope exists.

Proof Indeed, in the notation of (10.1), $\zeta$ changes the sign of $S_{0}$, which in turn changes the sign of $R_{1}$; by definition, this is $\zeta_{1}$.

Before we go on to discuss individual cases, we have a further general result about this construction; this refines Proposition 10.3. Suppose that $Q$ is a (finite) classical regular $d$-polytope, with group $\mathcal{G}$ generated by hyperplane reflexions $\left(S_{0}, \ldots, S_{d-1}\right)$. Then $P:=Q^{\zeta \varpi}$ has group generators $\left(S_{1},-S_{0} S_{2}, S_{3}, \ldots, S_{d-1}\right)$ (as usual, we take $o$ to be the centre of $Q)$. The mirror of $-S_{0} S_{2}$ is $\left(S_{0} \cap S_{2}\right)^{\perp}$, so that the Wythoff space of $P$ (containing its initial vertex) is

$$
\left(S_{0} S_{2}\right)^{\perp} \cap S_{3} \cap \cdots \cap S_{d-1}=\left(S_{0}^{\perp}+S_{2}^{\perp}\right) \cap S_{3} \cap \cdots \cap S_{d-1} .
$$

Now, if $u_{j}$ is a (unit) normal to $S_{j}$ for $j=0,2$, then

$$
S_{0}^{\perp}+S_{2}^{\perp}=\operatorname{lin}\left\{u_{0}, u_{2}\right\} .
$$

Since $u_{0} \in S_{3} \cap \cdots \cap S_{d-1}$ but $u_{2} \notin S_{3} \cap \cdots \cap S_{d-1}$, it follows that the Wythoff space of $P$ is actually $\operatorname{lin}\left\{u_{0}\right\}$. We have thus shown the following:

Theorem 10.4 If $Q$ is a finite classical regular d-polytope with group $\mathcal{G}(Q)=$ $\left\langle S_{0}, \ldots\right\rangle$, and if $u_{0}$ is the normal to the hyperplane $S_{0}$, then $u_{0}$ can be taken to be the initial vertex of $Q^{\zeta \varpi}$, if this polytope is non-degenerate.

Remark 10.5 In fact, Theorem 10.4 gives an easy criterion for degeneracy, when too few vertices would be provided by the images of $u_{0}$ under $\mathcal{G}(Q)$ compared to the index $\left[\mathcal{G}(Q):\left\langle S_{3}, \ldots, S_{d-1}\right\rangle\right]$. We shall see specific examples almost immediately.

We now look at each case separately.

\subsection{Simplex}

For the group $\mathcal{S}_{d+1}=\mathcal{G}(Q)$ of the regular simplex $Q=\alpha_{d}$ (the symmetric group of order $(d+1)$ !), as in earlier papers we work in the hyperplane $\mathbb{H}^{d}:=\{x=$ $\left.\left(\xi_{0}, \ldots, \xi_{d}\right) \mid \xi_{0}+\cdots+\xi_{d}=0\right\}$ in $\mathbb{E}^{d+1}$. Thus we can take the generators $\mathcal{G}(Q)$ to be transpositions of $\{0, \ldots, d\}$, and so, with $S_{j}:=(j j+1)$ for $j=0, \ldots, d-1$, we have

$$
R_{0}=\left(\begin{array}{ll}
1 & 2
\end{array}\right), \quad R_{1}=\left(\begin{array}{ll}
0 & 1
\end{array}\right)(23), \quad R_{j}=(j+1 j+2) \quad \text { for } j=2, \ldots, d-1 .
$$

The 3-face of $P=Q^{\varpi}$ is the polyhedron $\left\{4, \frac{6}{2,3}: \frac{5}{1,2} \mid 3\right\}$ of $[17,18]$, while the vertex-figure of $P$ is $\left\{3^{d-3}\right\} \#\{2\}$. It is easy to check the geometry here; note that the initial vertex of $P$ can be taken to be $\left(d-1, d-1,(-2)^{d-1}\right)$.

If we replace $Q$ by $Q^{\zeta}$, then we change the sign of $R_{1}$; that is, $\zeta \varpi=\varpi \zeta_{1}$. In view of Theorem 10.4 , the initial vertex of $P$ can be taken to be $\left(1,-1,0^{d-1}\right.$ ) (a normal 
vector to $S_{0}$ ), making it easy to check the geometry. We thus see that the original 3face is replaced by its double cover $\left\{4, \frac{6}{1,3} \mid 3\right\} \cong\{4,6 \mid 3\}$, one of the skew polyhedra of Coxeter [3]. The vertex-figure is now of the form $\left\{3^{d-3}\right\} \diamond\{2\}$, and the order of the group of $P$ is doubled to $2 \cdot(d+1)$ !.

Recalling that we have $d \geq 5$ here, we see that $\pi$ can only be applied to $P$ (in either case) when $d=5$. In the first case, the vertex-figure of $P$ can be written $\left\{\frac{6}{2,3}, 3\right.$ : $\left.\frac{4}{1,2}\right\} \cong\{6,3\}_{4}$. What then results is

$$
\left\{\left\{4, \frac{6}{2,3}: \frac{5}{1,2} \mid 3\right\},\left\{\frac{6}{2,3}, 3: \frac{4}{1,2}\right\}\right\}^{\pi}=\left\{\left\{4, \frac{4}{1,2} \mid 3\right\},\left\{\frac{4}{1,2}, 3: \frac{6}{2,3}\right\}: \frac{5}{1,2}\right\} .
$$

We have left the signifier for the Petrie polygon of the vertex-figure as an indication of its geometry (it is actually $\left\{\frac{4}{1,2}, 3: 3\right\} \#\{2\}$ ). In the other case, the resulting 4-polytope is the double cover of the 4-polytope of (10.2); we obtain

$$
\left\{\left\{4, \frac{6}{1,3} \mid 3\right\},\left\{\frac{6}{1,3}, 3: \frac{4}{1,2}\right\}\right\}^{\pi}=\left\{\left\{4, \frac{4}{1,2} \mid 3\right\},\left\{\frac{4}{1,2}, 3: \frac{6}{1,3}\right\}\right\} .
$$

This polytope is isomorphic to the universal $\{\{4,4 \mid 3\},\{4,3\}\}$; we shall meet it again in Sect. 12.

Remark 10.6 Since the appropriate entry in the Schläfli type of $\alpha_{6}^{\varpi}$ is even (it is 6, of course), it might be thought that $\pi$ would be applicable here as well. However, a little checking shows that the degeneracy of its putative edge-figure $\alpha_{4}^{\pi}$ is not rescued by blending with $\{2\}$.

We can now apply $\zeta$ to all these polytopes. With $\alpha_{d}^{\varpi}$ and its Petrial in case $d=5$ only, we obtain polytopes with twice as many vertices, and group of order $2 \cdot(d+1)$ !. In the other two cases, since the polytopes are already centrally symmetric, the group order remains the same. The details are moderately uninteresting, and so we shall say no more about them (although it might be worth the reader's while to verify that no nasty degeneracies occur).

\subsection{Cross-polytope}

Since the facet of the $d$-cross-polytope $Q=\beta_{d}$ is the $(d-1)$-simplex, much of what we have just said about the case of the simplex carries over directly. In view of $d \geq 5$, the 3 -face of $P=Q^{\varpi}$ is again $\left\{4, \frac{6}{2,3}: \frac{5}{1,2} \mid 3\right\}$, while its vertex-figure is now $\left\{3^{d-4}, 4\right\} \#\{2\}$. However, it helps in what we do next to write down the group generators explicitly. In terms of $x=\left(\xi_{1}, \ldots, \xi_{d}\right)$, we can express $R_{0}, \ldots, R_{d-3}$ as permutations of the coordinates, namely,

$$
R_{0}=(23), \quad R_{1}=(12)(34), \quad R_{j}=(j+2 j+3) \quad \text { for } j=2, \ldots, d-3,
$$

and

$$
R_{d-2}: x \mapsto\left(\xi_{1}, \ldots, \xi_{d-1},-\xi_{d}\right) .
$$

With initial vertex $\left(1,1,0^{d-2}\right)$, we see that the facet is $\left\{3^{d-2}\right\}^{\varpi}$ (as it must be); this is just a scaled and translated copy of the previous polytope in case $d-1$ instead of $d$ (and coordinate indices running from 1 to $d$ instead of 0 to $d-1$ ). 
Now $\zeta_{1}$ replaces $R_{1}$ by $-R_{1}$ (when $Q$ is replaced by $Q^{\zeta}$ ), so that the new $R_{1}$ is

$$
x \mapsto-\left(\xi_{2}, \xi_{1}, \xi_{4}, \xi_{3}, \xi_{5}, \ldots, \xi_{d}\right) .
$$

As we should by now expect, the effect of $\zeta_{1}$ is to replace the old 3 -face by $\left\{4, \frac{6}{1,3} \mid\right.$ $3\} \cong\{4,6 \mid 3\}$. The new vertex-figure is still of type $\left\{3^{d-3}, 4\right\} \#\{2\}$. Note that the new initial vertex is $\left(1,-1,0^{d-2}\right)$ (again, a normal to $S_{0}$ ); this time, we actually have as facet $\left\{3^{d-2}\right\}^{\zeta \varpi}=\left\{3^{d-2}\right\}^{\varpi \zeta_{1}}$ with the right vertices.

Once more, the Petrie operation $\pi$ is only permissible if $d=5$ (it is invalid if $d=6$ for the same reason that it fails in the case of the simplex $\alpha_{6}$ ). However, we encounter a pretty coincidence. The operations $\zeta_{1}$ and $\pi$ only affect $R_{1}$ and change it into

$$
\begin{aligned}
& R_{1}^{\zeta_{1}}: x \mapsto\left(-\xi_{2},-\xi_{1},-\xi_{4},-\xi_{3},-\xi_{5}\right), \\
& R_{1}^{\pi}: x \mapsto\left(\xi_{2}, \xi_{1}, \xi_{4}, \xi_{3},-\xi_{5}\right)
\end{aligned}
$$

respectively. We can easily see that $\left(R_{0}, R_{1}^{\zeta 1}, R_{2}, R_{3}\right)$ and $\left(R_{0}, R_{1}^{\pi}, R_{2}, R_{3}\right)$ are conjugate under the reflexion $T: x \mapsto\left(\xi_{1},-\xi_{2}, \xi_{3},-\xi_{4}, \xi_{5}\right)$, and so it follows that $\beta_{d}^{\zeta \varpi}=\beta_{d}^{\varpi \zeta_{1}}=\beta_{d}^{\varpi \pi} T$.

Naturally, we can apply $\zeta$ to both these polytopes (but again it is worth checking that nothing goes wrong).

\subsection{Cube}

We can take the generators of the group of the $d$-cube $\gamma_{d}$ to be

$$
S_{0}: x \mapsto\left(-\xi_{1}, \xi_{2}, \ldots, \xi_{d}\right)
$$

with $S_{j}$ the permutation $(j j+1)$ of the coordinates of $\mathbb{E}^{d}$ for $j=1, \ldots, d-1$. Thus the new generator $R_{1}$ of the group of $\gamma_{d}^{\varpi}$ is

$$
R_{1}: x \mapsto\left(-\xi_{1}, \xi_{3}, \xi_{2}, \xi_{4}, \ldots, \xi_{d}\right)
$$

The initial vertex can be taken to be $\left(0,1^{d-1}\right)$, the 3 -face is the polyhedron $\left\{6, \frac{6}{2,3}\right.$ : $\left.\frac{8}{1,3} \mid 3\right\}=\gamma_{4}^{\varpi}$ (see Remark 10.1), and the vertex-figure is $\alpha_{d-2} \#\{2\}$.

The Schläfli type of this polytope is $\left\{6,6,3^{d-4}\right\}$, and so we might expect the Petrie operation $\pi$ to be applicable if $d=5$ or 6 (as usual, we exclude the case $d=4$, which was already treated in [17]). However, it fails in case $d=6$ for the same reason as it did for the simplex. For $d=5$, we obtain the polytope

$$
\left\{\left\{6, \frac{4}{1,2} \mid 3\right\},\left\{\frac{4}{1,2}, 3: \frac{6}{2,3}\right\}: \frac{8}{1,3}\right\} .
$$

The facet is indeed as claimed, and not its quotient $\left\{6, \frac{4}{1,2}: \frac{5}{1,2} \mid 3\right\}$ of index 2 ; the 30 vertices of the initial facet are those of the whole polytope lying in the hyperplane $\xi_{1}+\xi_{2}-\xi_{3}-\xi_{4}+\xi_{5}=0$. There are 16 equivalent hyperplanes (orthogonal to diagonals of the 5 -cube), in accord with the calculation $2^{5} \cdot 5 ! / 2 \cdot 5 !=16$ for the number 
of facets. Note that the universal polytope of type $\{\{6,4 \mid 3\},\{4,3\}\}$ is infinite, as we shall see in Sect. 12, and so the Petrie polygon relation in the notation is needed to ensure finiteness.

Naturally, we can apply $\zeta$ to both these polytopes.

Last, we show that $\gamma_{d}^{\zeta \varpi}=\gamma_{d}^{\varpi \zeta_{1}}$ degenerates. To obtain this supposed polytope, we change the sign of $S_{0}$ above and hence the sign of $R_{1}$. An appeal to Theorem 10.4 gives $e_{1}=\left(1,0^{d-1}\right.$ ) (a normal to $S_{0}$ ) as the initial vertex; we thus obtain only $2 d$ vertices in all, rather than the $2^{d} \cdot d ! / 2 \cdot 2^{d-2} \cdot(d-2) !=2 d(d-1)$ expected from the index of the group of the vertex-figure. To bolster this argument, we see that the 2-face is a doubly covered triangle. Moreover, of course, the example cannot be rescued by a further application of $\zeta$ (changing the sign of $R_{0}$ ); one obtains 3 -faces which should be of type $\{6,6\}$, with 8 vertices and 4 hexagonal faces, an obvious contradiction. Again as we would expect, we cannot obtain genuine polytopes by applying $\pi$ to these non-polytopes (when $d=5$ ).

\subsection{Cubic tiling}

Apart from the free abelian apeirotopes, there are only two regular apeirotopes of full rank in $\mathbb{E}^{d}$ for $d \geq 5$, namely, the cubic tiling $\delta_{d+1}=\left\{4,3^{d-2}, 4\right\}$ and the result of applying $\kappa$ to it (that is, applying $\zeta$ to its vertex-figure $\beta_{d}=\left\{3^{d-2}, 4\right\}$ ). However, there is a connexion to the apeirotopes apeir $\beta_{d}$ and apeir $\beta_{d}^{\zeta}$. If the group of $\delta_{d+1}$ has generators $\left(S_{0}, \ldots, S_{d}\right)$ and $E$ is the reflexion in the line containing the initial edge, so that $E=\left(S_{2} \cdots S_{d}\right)^{d-1}$, then (in the notation of Sect. 5-see also [16]) the operation

$$
\kappa_{02}:\left(S_{0}, \ldots, S_{d}\right) \mapsto\left(S_{0} E, S_{1}, \ldots, S_{d}\right)=:\left(T_{0}, \ldots, T_{d}\right)
$$

yields the group $\left\langle T_{0}, \ldots, T_{d}\right\rangle$ of apeir $\beta_{d}$.

For $\delta_{d+1}^{\varpi}$, we can read off the information from what we have just done on the cube. Thus, it has facet $\gamma_{d}^{\varpi}$ and vertex-figure $\beta_{d-1} \#\{2\}$. For $\delta_{d+1}^{\kappa \varpi}$, we need to mention coordinates. The initial vertex of $\delta_{d+1}^{\varpi}$ can be taken to be $e_{1}=\left(1,0^{d-1}\right)$, the midpoint of the initial edge of the cubic tiling of side length 2; the corresponding first two reflexions are

$$
\begin{aligned}
& R_{0}: x \mapsto\left(\xi_{2}, \xi_{1}, \xi_{3}, \ldots, \xi_{d}\right), \\
& R_{1}: x \mapsto\left(2-\xi_{1}, \xi_{3}, \xi_{2}, \xi_{4}, \ldots, \xi_{d}\right)
\end{aligned}
$$

Applying $\kappa$ before $\varpi$ changes the sign of $R_{0}$, resulting in

$$
R_{1} \cdot\left(-R_{0}\right): x \mapsto\left(-\xi_{3}, \xi_{1}-2,-\xi_{2},-\xi_{4}, \ldots,-\xi_{d}\right),
$$

so that

$$
\left(R_{1} \cdot\left(-R_{0}\right)\right)^{3}: x \mapsto\left(\xi_{1}-2, \xi_{2}-2, \xi_{3}+2,-\xi_{4}, \ldots,-\xi_{d}\right) .
$$

With the same initial vertex $e_{1}$ as before, this tells us that the new 2-face is a helical apeirogon $\left\{\frac{3}{0,1}\right\}$ (the changes of sign in the last $d-3$ coordinates do not affect it). The case $d=4$, in effect investigated in [18], shows that the 3 -face is of type $\left\{\frac{3}{0,1}, \frac{6}{2,3}\right.$ : $\left.\frac{8}{1,3} \mid \frac{6}{2,3}\right\}$. 


\section{The First Gosset Class}

This section is devoted to a description of a remarkable family of regular polytopes and apeirotopes of nearly full rank, of which the basic examples are closely related to the uniform Gosset-Elte polytopes. (Uniformity is a somewhat weak condition; it only demands regular 2-faces, uniform facets and a symmetry group transitive on the vertices. The polytopes $r_{21}$ have regular facets-cross-polytopes $r_{11}$ and simplices $r_{20}$ - and so are often referred to as semi-regular.) In fact, the sole example of these which is original to Elte [11] (that is, not found by Gosset [12]) is $1_{42}$, which does not contribute to our family (hence we drop reference to Elte from now on). We naturally also include the apeirotopes in this family here; however, we postpone discussion of some other extensions to Sect. 13.

The source of the family is the Petrie contraction $Q:=\{3,3,3\}^{\varpi}=\left\{4, \frac{6}{2,3}: \frac{5}{1,2} \mid\right.$ $3\} \cong\{4,6: 5 \mid 3\}$ of the 4-simplex, a four-dimensional regular polyhedron that was first described in [20]; it was briefly discussed in [17], but we did not specify its group until [18]. (In [19], it is shown that a sufficient geometric description of this polyhedron is $\left\{4, \frac{6}{2,3} \mid 3\right\}$. However, we shall keep to the earlier notation here, because this also indicates the abstract type.) The group $\mathcal{G}(Q)$ of $Q$ is $[3,3,3] \cong S_{5}$; this is fairly clear from the construction, but we give a brief extra explanation in abstract terms.

Because $Q$ slots in at different places in polytopes of our family, initially we adopt a neutral notation $(S, T, U)$ for the generators of $\mathcal{G}(Q)$. Because $Q$ is obtained by Petrie contraction from the 4-simplex, as described in Sect. 10, if we write $(X, S, Y, U)$ for the natural generators of $[3,3,3]$, then $T:=X Y$ yields $\mathcal{G}(Q)$. From this, it is purely routine to verify that $\mathcal{G}(Q)=\langle S, T, U\rangle$ indeed satisfies the relations implied by the notation $\{4,6: 5 \mid 3\}$.

It is only slightly more difficult to establish the relationship in reverse; in principle, this was shown in [17]. If we are given involutions $S, T, U$ satisfying

$$
(S T)^{4}=(T U)^{6}=(S T U)^{5}=(S T U T)^{3}=I, \quad S \rightleftharpoons U,
$$

where the relation $\rightleftharpoons$ means 'commutes with', then we define

$$
X:=(T U)^{3}=(U T)^{3}, \quad Y:=T X .
$$

Most of the relations for [3,3,3] are easily recovered; the trickiest is $(S X)^{3}=I$, for which we need to use (twice) $(S T U T)^{3}=I$ (which implies that TUTSTUT= $S T U T S)$ and finally $(S U T)^{5}=I$. We suppress the rather uninteresting details.

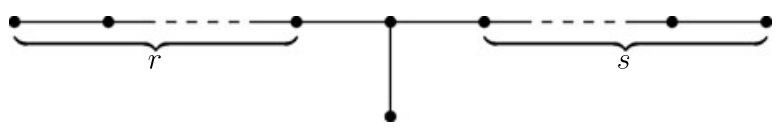

We now move on to the general case. The preceding diagram is that of the finite or discrete Euclidean Coxeter group [ $\left.3^{r, s, 1}\right]$; as usual, the nodes represent the involutory generators (which are hyperplane reflexions), and two nodes are joined by a branch or not according as the product of the corresponding pair of generators has period 3 
or 2. Recall that $\left[3^{r, s, 1}\right]$ is a finite or Euclidean reflexion group just when

$$
\frac{1}{r+1}+\frac{1}{s+1} \geq \frac{1}{2} \quad \Leftrightarrow \quad(r-1)(s-1) \leq 4
$$

with equality giving the Euclidean cases; see [5, p. 31] for a neat proof of this.

The infinite cases are depicted below; of course, the finite cases are obtained by deleting nodes at the beginning or end of the horizontal string. The labelling $X, S, Y, U$ is as we have just discussed. We demand that $r \geq 1$ (since the case $r=0$ was treated in Sect. 10) and $s \geq 2$. Thus $R$ is always present; however, $V$ may be absent (as in the third diagram).
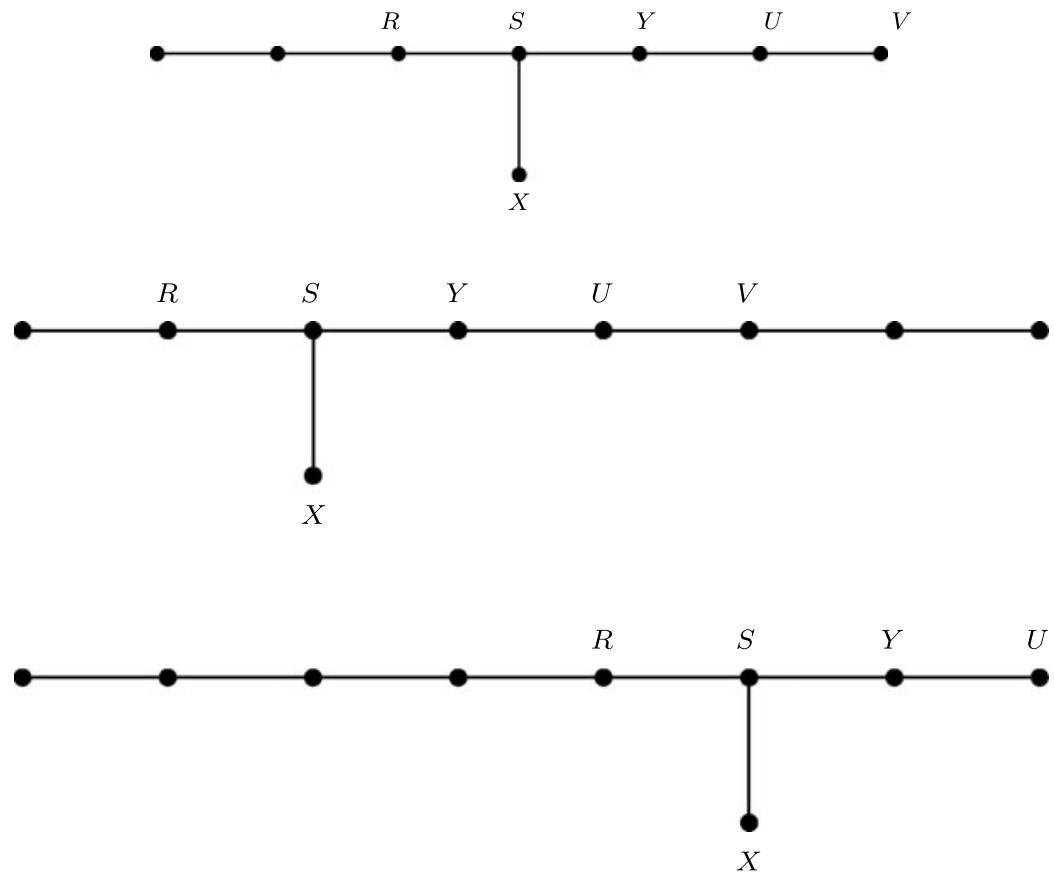

We first need to see how $R$ and $V$ interact with the new generator $T=X Y$. Since $R \rightleftharpoons X, Y$, we see that $R \rightleftharpoons T$, and it follows that $\langle R, S, T\rangle \cong[3,4]$ in the natural way. Next, since $V \rightleftharpoons X, Y$, we see that $V \rightleftharpoons T$. However,

$$
V U T=V U Y \cdot X \quad \Rightarrow \quad(V U T)^{4}=I
$$

and since (as we already know) $T U$ has period 6, we see that $\langle T, U, V\rangle$ is the group of a polyhedron of type $\{6,3: 4\}$. In fact (comparing [17]), it is $\{3,3\} \#\{2\}=\left\{\frac{6}{2,3}, 3\right.$ : $\left.\frac{4}{1,2}\right\} \cong\{6,3: 4\}$.

We are now in a position to describe the polytopes in this family.

Proposition 11.1 For each $r \geq 0$ and $s \geq 2$ with $(r-1)(s-1) \leq 4$, there is a regular polytope (or apeirotope) $G_{r s}$ of nearly full rank with the following properties: 
- it has rank $r+s+1$,

- its group $\mathcal{G}_{r s}$ is $\left[3^{r, s, 1}\right]$,

- it has the same vertices as the Gosset polytope (or apeirotope) $r_{s 1}$,

- its $(r+2)$-faces are $(r+2)$-cross-polytopes $\beta_{r+2}$,

- its $(s+1)$-cofaces are Petrie contractions $\alpha_{s+2}^{\varpi}$ of $(s+2)$-simplices.

In term of the original diagram, the group generators are $(\ldots, R, S, X Y, U, V, \ldots)$ (with $V$ omitted if $s=2$ ). The faces and cofaces given by Theorem 11.1 define $G_{r s}$ as an abstract regular polytope, when we bear in mind what the 3- and 4-faces of $\alpha_{s+2}^{\varpi}$ are. Thus $G_{r s} \cong\left\{3^{r}, 4,6,3^{s-2}\right\} /\langle\langle\mathrm{J}, \mathrm{K}, \mathrm{L}\rangle\rangle$, with $\mathrm{J}, \mathrm{K}, \mathrm{L}$ the relators

$$
\begin{aligned}
& \mathrm{J}:=(r(r+1)(r+2))^{5}, \quad \mathrm{~K}:=(r(r+1)(r+2)(r+1))^{3}, \\
& \mathrm{~L}:=((r+1)(r+2)(r+3))^{4} ;
\end{aligned}
$$

the last relator is absent if $s=2$.

Let us say a little more about the case $s=2$. The Gosset polytope $r_{21}$ has two kinds of facet, namely, cross-polytopes and simplices (of dimension $r+3$ ). These meet two cross-polytopes and one simplex around each $(r+1)$-face. We can think of the facets of the cross-polytopes 2-coloured black and white and those of the simplices coloured white, with two cross-polytopes abutting on black facets, and crosspolytopes and simplices abutting on white ones. The facets of $G_{r 2}$ are then the diametral $(r+2)$-cross-polytopes of these cross-polytopal facets of $r_{21}$, and we see from this description that they do indeed meet in pairs on each of the simplicial $(r+1)$ faces of $r_{21}$.

We can now apply the operation $\zeta$ to all the finite polytopes in the family; the resulting polytopes are not particularly interesting, and so we shall say no more about them than that three (namely, $G_{42}^{\zeta}, G_{15}^{\zeta}, G_{23}^{\zeta}$ ) are vertex-figures of apeirotopes-see below. On the other hand, applications of $\pi$ (where appropriate) lead to some interesting polytopes. In fact, $\pi$ can only apply to $G_{r s}$ when $s=2$ or 3; see Remark 11.2. Moreover, if $s=2$, the only valid case is $r=0$, so we have a polyhedron; we have dealt with this in [17].

Remark 11.2 Observe that the Petrie operation $\pi$ does not apply when $s=4$, in spite of the fact that the relevant entry 6 in the Schläfli symbol is even. Indeed, by Theorem 5.7, the operation fails because of the hole $\{3\}$ in the face $G_{02}$.

Thus $s=3$ is the only productive case in the present context. We first treat the subcase $r=0$. Going back to the way we constructed the group from $\left[3^{0,3,1}\right]$ (that is, in the first diagram, with no left branch, and only $V$ in the right branch), we see that the new group has generators $(S, T V, U, V)=(S, X Y V, U, V)$. A little work shows that the facet of the resulting polytope is $\left\{4, \frac{4}{1,2} \mid 3\right\} \cong\{4,4 \mid 3\}$, while the vertex-figure is $\left\{\frac{4}{1,2}, 3: \frac{6}{2,3}\right\} \cong\{4,3\}$. However, this is not universal of type $\{\{4,4 \mid 3\},\{4,3\}\}$, as we shall shortly see. Indeed, if we anticipate a little, we find that we actually have

$$
G_{03}^{\pi}=\left\{\left\{4, \frac{4}{1,2} \mid 3\right\},\left\{\frac{4}{1,2}, 3: \frac{6}{2,3}\right\}: \frac{5}{1,2}\right\} \cong\{4,4,3\} /\left\langle(0121)^{3},(0123)^{5}\right\rangle .
$$


In turn, we can use these as successive vertex-figures, to construct $G_{r 3}^{\pi}$ for $r=$ $1,2,3$, the last being an apeirotope in $\mathbb{E}^{7}$. The case $r=1$ is worth describing in some detail. Writing $\left(R_{0}, \ldots, R_{4}\right):=(R, S, X Y V, U, V)$ with the notation of the original diagram, in view of the way that $G_{11}^{\pi}$ is derived we can take

$$
\begin{aligned}
& R_{0}: x \mapsto\left(-\xi_{2},-\xi_{1}, \xi_{3}, \xi_{4}, \xi_{5}, \xi_{6}\right), \\
& R_{1}: x \mapsto\left(\xi_{1}, \xi_{3}, \xi_{2}, \xi_{4}, \xi_{5}, \xi_{6}\right), \\
& R_{2}: x \mapsto\left(\xi_{2}, \xi_{1}, \xi_{4}, \xi_{3}, \xi_{6}, \xi_{5}\right), \\
& R_{3}: x \mapsto\left(\xi_{1}, \xi_{2}, \xi_{3}, \xi_{5}, \xi_{4}, \xi_{6}\right), \\
& R_{4}: x \mapsto\left(\xi_{1}, \xi_{2}, \xi_{3}, \xi_{4}, \xi_{6}, \xi_{5}\right) .
\end{aligned}
$$

The vertices are those of the half-cube $h \gamma_{6}$, which we write as $c_{j k \ldots}=$ $\left(\varepsilon_{1}, \varepsilon_{2}, \varepsilon_{3}, \varepsilon_{4}, \varepsilon_{5}, \varepsilon_{6}\right)$, with each $\varepsilon_{i}= \pm 1$, to indicate that $\varepsilon_{i}=-1$ precisely for $i=j, k, \ldots$ (with $c:=c_{\emptyset}$ ); thus, for example, $c_{1246}=(-1,-1,1,-1,1,-1)$. The initial vertex is $c$, which is joined to $c_{12}$ by the initial edge; the initial 2 -face then has the additional vertex $c_{13}$. A little work shows that the initial 3-face (which is an octahedron) has opposite pairs of vertices $c, c_{1234}: c_{12}, c_{34}: c_{13}, c_{24}$. Finally, the vertexfigure of the initial facet consists of all $c_{j k}$ with $j=1,4,5$ and $k=2,3$, 6, with square faces given by two out of three of the choices for $j$ and $k$. The facet itself is centrally symmetric about the origin $o$. We thus see that the facet $\left\{\{3,4\},\left\{4, \frac{4}{1,2} \mid 3\right\}\right\}$ is, in fact, the universal $\{\{3,4\},\{4,4 \mid 3\}\}$ with 20 vertices and 30 octahedral 3 -faces.

However, the vertex-figure cannot be the dual of this, in spite of the apparent symmetry of the diagram, because it only has 15 vertices and 10 toric 3 -faces. Since the Petrie polygon of $\{\{4,4 \mid 3\},\{4,3\}\}$ has period 10 , while that of the vertex-figure has period 5, we see that it is indeed obtained by imposing the extra relator $(0123)^{5}$ on the group of the former. In other words, we conclude that

$$
\left.G_{13}^{\pi} \cong\{3,4,4,3\} / \|(1232)^{3},(1234)^{5}\right\rangle,
$$

when we bear in mind that we can recover the original group $\left[3^{1,3,1}\right]$ by reversing the various operations which led to $G_{13}^{\pi}$.

Our polytope $G_{13}^{\pi}$ has no dual, because its vertex-figure does not. However, if we change the sign of $R_{2}$, and so replace it by $-R_{2}=R_{2}^{\perp}$, and reverse the order of the generators, namely,

$$
\left(R_{0}, \ldots, R_{4}\right) \mapsto\left(R_{4}, R_{3},-R_{2}, R_{1}, R_{0}\right)=:\left(S_{0}, \ldots, S_{4}\right),
$$

then we have the group $\left\langle S_{0}, \ldots, S_{4}\right\rangle$ of another copy of $G_{13}^{\pi}$. Its vertices consist of those of the 'complementary' copy of $h \gamma_{6}$ in the 6-cube $\gamma_{6}$; the initial vertex $c_{145}$ (or $c_{236}$ ) corresponds in an obvious way to the initial facet of the original.

We shall say little about $G_{r 3}^{\pi}$ for $r=2$ or 3 . The former has group $\left[3^{2,3,1}\right]$ and the vertices of $2_{31}$. The latter has group $\left[3^{3,3,1}\right]$ and the vertices of the apeirotope $3_{31}$; perhaps one remarkable feature is that its facets are also apeirotopes, of the type $A_{6}$ (with group $\left[3^{2,2,2}\right] \rtimes \mathcal{C}_{2}$ ) which we shall discuss in the next Sect. 12 .

We have three apeirotopes $G_{52}, G_{25}$ and $G_{33}$, as well as the Petrial $G_{33}^{\pi}$ of the last, to each of which the operation $\kappa$ is applicable; note that all the vertex-figures 
are centrally symmetric, and none is blended. The 2 -faces are hexagons $\{6\}$, and it should not be a surprise that the facets are apeirotopes of rank deficit 2.

There are also polytopes or apeirotopes related to the Gosset polytopes $r_{22}$, but again we shall leave them until Sect. 13.

\section{Twisting}

In defining twisting in Sect. 5, we remarked that we would find it convenient to allow inner automorphisms as twists. Let us begin by illustrating the latter point. Consider the Petrie contraction $\left\{3^{d-1}\right\}^{\varpi}$ of the $d$-simplex $\alpha_{d}=\left\{3^{d-1}\right\}$. In terms of the transpositions $(j k)$ in the symmetric group $S_{d+1}$, the generators of its group are

$$
\begin{aligned}
& R_{0}:\left(\begin{array}{ll}
1 & 2
\end{array}\right), \\
& R_{1}:\left(\begin{array}{ll}
0 & 1
\end{array}\right)(23), \\
& R_{j}:(j+1 j+2) \text { for } j=2, \ldots, d-2 .
\end{aligned}
$$

In the spirit of [18], we can also represent the group by a diagram, acted upon by an (inner) automorphism $R_{1}$, as follows:

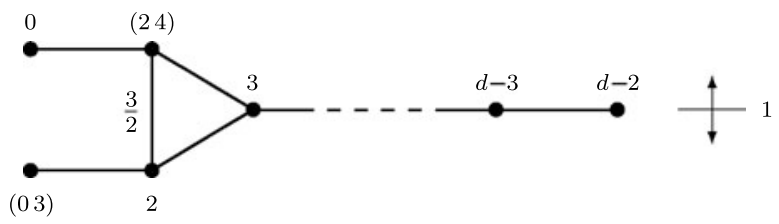

As usual, a number $j$ against a node refers to the generator $R_{j}$; we have similarly indicated the twist $R_{1}$ and two other relevant transpositions.

On the other hand, if we consider instead $\alpha_{d}^{\zeta \varpi}$, we change the sign of the reflexion $R_{1}$ to obtain $-R_{1}=-\left(\begin{array}{ll}0 & 1\end{array}\right)$ (2 3$)$ with an obvious meaning. Now $-R_{1}$ twists the same diagram, but with the mark $\frac{3}{2}$ removed and a mark 2 placed in the triangle.

In all cases, our basic examples will be obtained by twisting diagrams. We distinguish four kinds of twist. First, a twist can be outer or inner, according as the induced automorphism is outer or inner. Second, while a twist must permute the hyperplane mirrors of the diagram reflexions, it need not permute the corresponding (unit) normal vectors to those mirrors-it may change some signs, while preserving others. If the normals can be chosen to be permuted by the twist, then we it proper; otherwise the twist is improper.

Referring to the examples just mentioned, $R_{1}$ is a proper inner twist, while $-R_{1}$ is an improper outer one.

We begin the systematic investigation with a crucial observation, bearing in mind that we are concerned here with polytopes of nearly full rank. This is that, if we do not allow redundant generators, then the only diagrams which can be twisted to yield 
unblended regular polytopes of nearly full rank at least 4 are those of the form

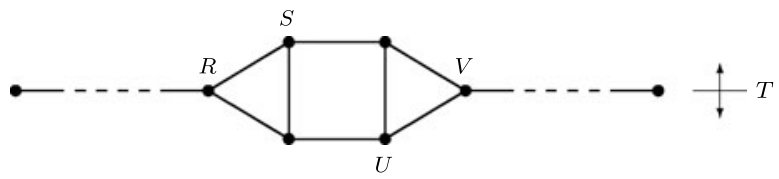

Branches may carry marks, and either vertical branch may be absent. The left and right branches form strings, and either may be absent (including the nodes $R$ or $V$ ). The twist is denoted by $T$. We shall not prove this claim formally, but we observe that each twist contributes 1 to the rank, while each node dropped from the generating set (and thus obtained by applying a twist to another node) lowers the rank by 1 . With two commuting twists we obtain a square sub-diagram (with diagonals-but here we will be obliged to introduce redundant generators if we wish to preserve the symmetry of the whole diagram), whereas two non-commuting twists (which must correspond to adjacent generating reflexions) will act on a single circuit; in neither case can we extend the diagram non-redundantly by further branches. With a single twist, we just have the picture above.

Remark 12.1 Note that basic blends with the segment $\{2\}$ can be obtained by twisting diagrams beginning with the right triangle.

Remark 12.2 Cases with redundant generators will be discussed at the end of the section.

We begin with an infinite family which is already known. In [16], we described the facet of the regular apeirotope $\delta_{d+1}^{\kappa}$, noting that it was full-dimensional and hence (in our present terms) of nearly full rank. In fact, of course, this facet must be $\gamma_{d}^{\kappa}$, as briefly described in Sect. 9. It is derivable by an improper outer twist of the diagram

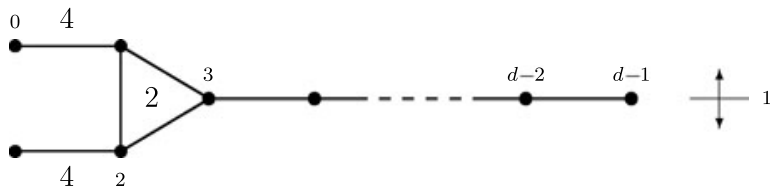

We have labelled the triangle rather than one of its edges; either of the two slanting edges can carry the label $\frac{3}{2}$, but the twist changes which is so labelled.

This diagram may at first glance seem impossible, since the four leftmost nodes already determine an infinite group. However, recalling the derivation of the apeirotope from $\delta_{d+1}$, it is easy to write down the generating reflexions and check that they do indeed correspond to the diagram (with twist). Observe also that we can extend the diagram to the right by a further branch labelled 4 , to give a diagram corresponding to $\delta_{d+1}^{\kappa}$ itself.

At this point, it is appropriate to correct a mistake in [18, p. 256]. In the discussion of the class $\mathcal{A}_{1}(2,4,2,3)$, we have the case $d=4$ of this apeirotope. In the (second) diagram on that page, and in the line immediately following, $\left\{4, \frac{6}{1,3} \mid 3\right\}$ occurs instead of what should be $\left\{4, \frac{6}{1,3} \mid 4\right\}$; compare the list on p. 235 of applications of $\kappa$ 
to the finite crystallographic polyhedra. In addition, the last sentence of the penultimate paragraph on p. 257 is misplaced since it refers to $\left\{\left\{4, \frac{6}{1,3} \mid 4\right\},\left\{\frac{6}{1,3}, 3: \frac{4}{1,2}\right\}\right\}$; this is indeed the facet of the four-dimensional apeirotope of full rank designated $\{4,6,3,4\}^{(s)}$ in the table of [16, p. 33].

There are further infinite families of diagrams which exhibit the same phenomenon, namely, having a proper subdiagram of an infinite group. We first have

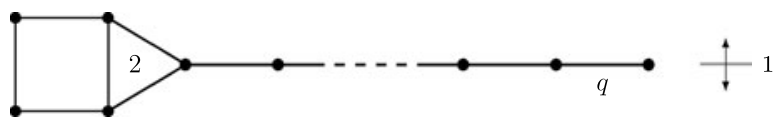

with $q=3$ or 4 . In effect, we have two families here, of which the facets of the second form the first. Once again, the corresponding apeirotopes are already known; they are $\alpha_{d}^{\kappa}$ or $\beta_{d}^{\kappa}$, according as $q=3$ or 4 .

In the notation of [4, Table IV] or [24, Table 3B2], the groups are $\mathcal{P}_{d+1} \times \mathcal{C}_{2}$ or $\mathcal{S}_{d+1}$. To obtain a presentation for the groups in terms of the given generators, we need to consider the Petrial of the 4-face. We should not be surprised to find that it is derived from the diagram of the unmarked pentagon with (now) a proper outer twist:

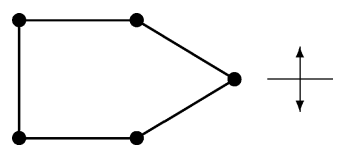

The Petrial is thus $\left\{\left\{6, \frac{4}{1,2} \mid 3\right\},\left\{\frac{4}{1,2}, 3: \frac{6}{1,3}\right\}\right\} \cong\{\{6,4 \mid 3\},\{4,3\}\}$, the universal polytope. Reversing $\pi$ (that is, applying it again) leads to the description of the original 4-face as $\left\{\left\{6, \frac{6}{1,3} \mid 3\right\},\left\{\frac{6}{1,3}, 3: \frac{4}{1,2}\right\}\right\} \cong\{\{6,6 \mid 3\},\{6,3: 4\}\}$, which is also universal. As we have seen earlier, the general notation concatenating the facet and vertex-figure can get rather clumsy; it is so here also, which leads to our using the alternative notation

$$
\left.\left\{6,6,3^{d-3}\right\} /\left\langle(0121)^{3},(123)^{4}\right\rangle, \quad\left\{6,6,3^{d-4}, 4\right\} \|(0121)^{3},(123)^{4}\right\rangle,
$$

respectively, for the two apeirotopes. It remains to remark that the respective vertexfigures are $\alpha_{d-1} \diamond\{2\}$ with the vertices of the cross-polytope $\beta_{d}$, and $\beta_{d-1}^{\zeta}$.

Note that the Petrie operation does not apply to the 6-face, as an appeal to Theorem 5.7 shows.

For the next four families, it is convenient to consider all cases together in order not to have to repeat calculations with shifts of indices or changes of dimension, and so we work with the diagram

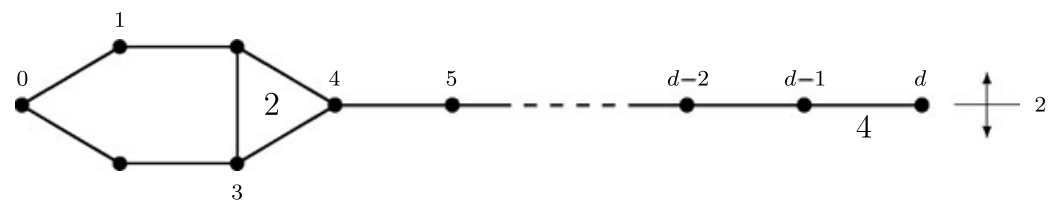

We then truncate the first or last nodes (or both) to obtain the other three families. 
As in Sect. 10, we work in $\mathbb{E}^{d+1}$ and its hyperplane

$$
\mathbb{H}:=\left\{x=\left(\xi_{0}, \ldots, \xi_{d}\right) \in \mathbb{E}^{d+1} \mid \xi_{0}+\cdots+\xi_{d}=0\right\} ;
$$

the generators of the whole group can be taken to be

$$
\begin{aligned}
& R_{0}: x \mapsto\left(1+\xi_{1},-1+\xi_{0}, \xi_{2}, \ldots, \xi_{d}\right), \\
& R_{1}:\left(\begin{array}{ll}
0 & 2
\end{array}\right) \text {, } \\
& R_{2}:-\left(\begin{array}{ll}
0 & 1
\end{array}\right)\left(\begin{array}{ll}
2 & 3
\end{array}\right) \text {, } \\
& R_{j}:(j j+1) \text { for } j=3, \ldots, d-1 \text {, } \\
& R_{d}: x \mapsto\left(\xi_{0}, \ldots, \xi_{d-1},-\xi_{d}\right) .
\end{aligned}
$$

As before, by -(l $\left.\begin{array}{ll}0 & 1\end{array}\right)\left(\begin{array}{l}2 \\ 2\end{array}\right)$ we mean the product of the transpositions composed with changing all signs; this twist $R_{2}$ is outer but improper. It is not hard to see that the facet has vertex-set $\mathbb{H} \cap \mathbb{Z}^{d+1}$, while that of the whole apeirotope is $\left\{\left(v_{0}, \ldots, v_{d}\right) \in\right.$ $\mathbb{Z}^{d+1} \mid v_{0}+\cdots+v_{d}$ even $\}$. Perhaps surprisingly, these are the same vertex-sets as in the previous case with $q=4$ (up to a change in rank and dimension); indeed, the groups are the same as well.

Remark 12.3 In the case $d=4$ (that is, in $\mathbb{E}^{5}$ ), the mark 4 on the end branch transfers to the slanting branches of the triangle (and the inner mark of the triangle changes to (3), in the notation of $[24,(9 D 8)])$.

We obtain the presentations of the groups (in terms of the given generators) as before. For the general case, we apply the Petrie operation to the 5-face, with the initial hexagon as diagram. In view of the previous case, we should not be surprised that the new diagram is an unmarked hexagon with a proper outer twist:

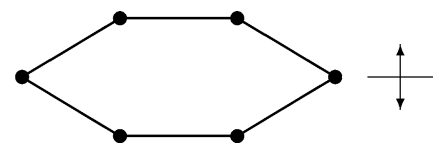

The corresponding apeirotope has facets $\left\{\{3,4\},\left\{4, \frac{4}{1,2} \mid 3\right\}\right\}$ and its dual vertexfigures $\left\{\left\{4, \frac{4}{1,2} \mid 3\right\},\left\{\frac{4}{1,2}, 3: \frac{6}{1,3}\right\}\right\}$ (we insert the mark $\frac{6}{1,3}$ for the Petrie polygon to emphasize that the corresponding polyhedron is $\{4,3\} \#\{2\}$ rather than $\left\{\frac{4}{1,2}, 3\right.$ : $3\} \#\{2\})$. Once more, the standard notation concatenating facet and vertex-figure is too clumsy; much better is merely to observe that it is isomorphic to the polytope $\{3,4,4,3\} /\left\langle\left\langle(1232)^{3}\right\rangle\right\rangle$ (we could perhaps write it as $\left\{\{3,4\},\left\{4, \frac{4}{1,2} \mid 3\right\},\left\{\frac{4}{1,2}, 3\right\}\right\}$ with little fear of confusion). As the notation suggests, this apeirotope is universal with the given facet and vertex-figure. Reversing $\pi$ shows the original 5 -face to be of type $\{3,4,6,3\} /\left\langle\left\langle(1232)^{3},(234)^{4}\right\rangle\right\rangle$. Of course, with rank $d>5$, the type is then $\left\{3,4,6,3^{d-5}, q\right\} /\left\langle\left\langle(1232)^{3},(234)^{4}\right\rangle\right\rangle$, with $q=3$ or 4 as appropriate.

Remark 12.4 We cannot extend this hexagonal diagram by an additional branch. The normal vector to the hyperplane corresponding to the (next) new node is orthogonal 
to those of the disjoint five nodes of the hexagon, and therefore to the sixth also, a contradiction.

Note that the Petrie operation does not apply to the 6-face; once again, we appeal to Theorem 5.7.

The Petrial in the rank 4 special case of Remark 12.3 is a little different. We obtain a proper but inner twist, on a diagram which may be written

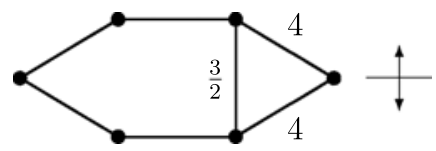

The facet here is now finite, of type $\left\{\{3,4\},\left\{4, \frac{6}{2,3}: \frac{5}{1,2} \mid 3\right\}\right\}$, while the edge-figure is $\left\{\frac{6}{2,3}, 4: \frac{6}{1,3}\right\}=\{3,4\} \#\{2\}$.

We have implicitly described the facet, vertex-figure and intermediate section (facet of vertex-figure) above, and the cases to which the Petrie operation $\pi$ applies. There remains to discuss the effects of applying $\kappa$ (or $\zeta$ to the vertex-figure). It was seen in $\left[18\right.$, Sect. 8] that $\left\{4, \frac{6}{1,3} \mid 3\right\}^{\zeta}=\left\{\frac{4}{1,2}, \frac{6}{1,3}: \frac{5}{1,2}\right\} \cong\{4,6: 5\}$. Moreover, we noted in Sect. 9 that $\{3,4\}^{\kappa}=\left\{6, \frac{4}{1,2} \mid 4\right\}$, a Petrie-Coxeter sponge. Thus the new abstract description in the general case is $\left\{6,4,6,3^{d-5}, q\right\} /\left\langle\left\langle(0121)^{4},(123)^{5},(234)^{4}\right\rangle\right\rangle$, with $q=3$ allowed if $d=5$. In the special case of Remark 12.3, we need to know that $\left\{4, \frac{6}{2,3}: \frac{5}{1,2} \mid 3\right\}^{\zeta}=\left\{\frac{4}{1,2}, \frac{6}{2,3}: \frac{10}{1,3} \mid \frac{6}{1,3}\right\}$; everything else goes through with no problems. Finally, $\kappa$ also applies when the diagram is the unmarked hexagon; the new 3 -face is again $\left\{6, \frac{4}{1,2} \mid 4\right\}$, and the new vertex-figure is isomorphic (but not congruent) to the old one.

In the spirit of [18] again, we can also derive the polytopes of Sects. 10 and 11 from diagrams, acted upon by a proper inner automorphism $T$. The crucial centre part of the diagram is

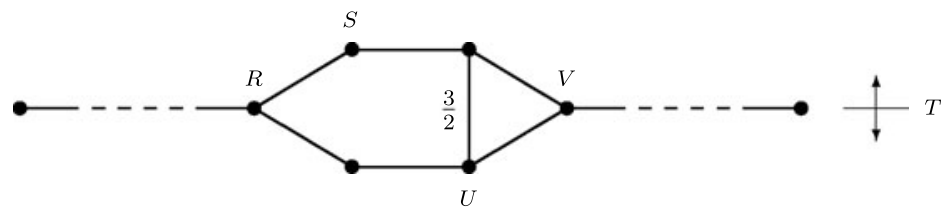

We will have $r \geq 2$ here, because we have already dealt with the cases $r=0$ and 1 earlier in the section (as two of the set of four families considered together). The notation carries over from that of Sect. 11.

In a similar way, the polytope $G_{13}^{\pi}$ described in the previous section can be derived by an improper inner twist from the diagram of a hexagon carrying a centre mark 2 (but otherwise unmarked). Note that the situation here is not the same as that in the case $\ell=2$ of [21, (39)], where an outer automorphism was applied to this hexagonal diagram.

We have already observed that the polytopes $G_{r s}$ do not have (geometric) duals. Nevertheless, we can remove the right branch from the diagram (beginning with the node $V$ ) and reverse it, to obtain the diagram below. The mark 2 on the circuit indicates as before that one of the branches - but not the vertical one-is to be thought 
of as marked $\frac{3}{2}$.

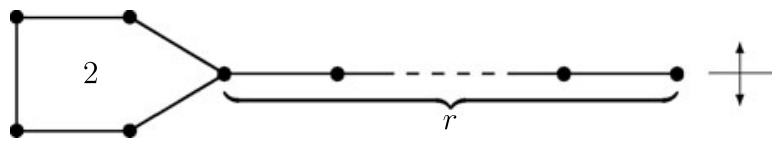

The group specified by the diagram is, again as before, $\left[3^{r, 2,1}\right]$, so that we must have $r \leq 5$, with $r=5$ the infinite case. We can then, potentially at least, apply an improper twist, to obtain a regular polytope $P_{n}$ of rank $n=r+3$.

However, something rather unexpected happens, in that we do not obtain the full family as might be expected. For $r=1$, we have the Petrial $P_{4}=\gamma_{5}^{\varpi \pi}$ of the Petrie contraction of the 5-cube $\gamma_{5}$, whose 80 vertices are the mid-points of the edges of $\gamma_{5}$. Its facets $\left\{6, \frac{4}{1,2} \mid 3\right\}$ (with 30 vertices) lie in central sections of $\gamma_{5}$, an important implication of which is that $P_{n-1}$ must be a central section of $P_{n}$ for $n \geq 5$, if the latter polytope exists. We immediately conclude-if for no other reason-that we cannot actually have an apeirotope $P_{8}$ derived from $\left[3^{5,2,1}\right]$.

For $r=2$, we obtain $P_{5}$, whose $270=27 \cdot 10$ vertices are those of the difference body $D T=T-T$ (Minkowski sum) of the Gosset polytope $T=2_{21}$; each vertex is uniquely expressible as, say, a sum $x+y$ of an $x \in \operatorname{vert}(-T)$ and $y \in \operatorname{vert} F_{x}$, with $F_{x}$ the facet $\beta_{5}$ of $T$ corresponding to $x$. Indeed, these 270 vertices are those of a central section of $3_{21}$ half-way between two opposite vertices; the 756 vertices of $P_{6}$ are all the mid-points of edges of $3_{21}$. In ranks 4 and 5, the twist is outer, while in rank 6 it is now inner; essentially, the twist involves the central inversion $-I$.

So far, there are no problems, but it is worth pointing out that, for rank 6 , we have an obvious vertex-figure $\gamma_{5} \diamond\{2\}$, with the vertices of $h \gamma_{5} \times \alpha_{1}$. However, the construction breaks down in rank 7. Calculations show that $P_{7}$ would have the 2160 vertices of 241 , and would share its group [ $\left.3^{4,2,1}\right]$. But now the vertex-figure would have the whole symmetry group $\left[3^{4,1,1}\right]$ of the half-cube $h \gamma_{7}$, rather than that of $\gamma_{6} \diamond\{2\}$. It follows that $P_{7}$ degenerates; from the group order, it should actually have $192 \cdot 10 ! / 2^{6} \cdot 6 !=15120$ vertices, so that the vertices collapse in sevens (corresponding, of course, to the seven ways of inscribing $\gamma_{6} \diamond\{2\}$ in $h \gamma_{7}$ ).

We should point out that $P_{7}$ was encountered in [16, Sect. 10] as the supposed facet of the second non-existent regular polytope of full rank (its group coinciding, as we have seen, with the whole group $\left[3^{4,2,1}\right]$ ). In fact, we now see that even this 'facet' was degenerate, let alone the whole 'polytope'.

Of course, we can now apply $\zeta$ to each of these examples $P_{n}$ for $n \leq 6$.

\section{The Second Gosset Class}

There remains the Coxeter diagram of the group $\left[3^{r, 2,2}\right]$ acted on by a proper outer twist, namely,

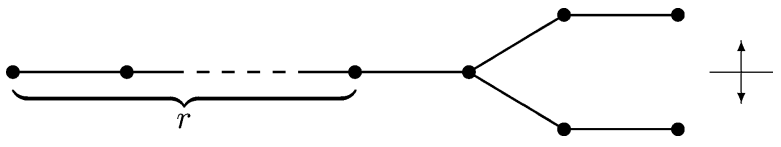


The case $r=0$ is the group $\left[3^{4}\right] \cong \mathcal{S}_{6}$ of the 5-simplex, which we have already encountered. Since the group is infinite for $r=2$, the only relevant cases are $r=0,1,2$. The twist acts as indicated in the picture. The case $r=0$ is the universal polytope

$$
\left\{\{3,4\},\left\{4, \frac{4}{1,2} \mid 3\right\}\right\} \cong\{\{3,4\},\{4,4 \mid 3\}\}=\{4,4,3\} /\left\langle(1232)^{3} \rrbracket .\right.
$$

In general, the facet is the cross-polytope $\beta_{r+3}$, for $r=1,2$, the vertex-figure is the case $r-1$, and the whole polytope (or apeirotope) $J_{r+4}$ (say $-r+4$ is the rank) is then universal with this facet and vertex-figure. Its vertex-set is the same as that of the Gosset polytope or honeycomb $r_{22}$, and each of these polytopes has a dual.

The case $r=2$ is of some interest because it is a sponge in $\mathbb{E}^{6}$, analogous to the Petrie-Coxeter apeirohedra in $\mathbb{E}^{3}$. The Gosset polytope $T=2_{21}$ and its negative $-T$ tile $\mathbb{E}^{6}$ by translates. On a simplicial facet $\alpha_{5}$, a translate of $T$ meets another translate of $T$ (and similarly for $-T$ ), while on a cross-polytope facet $\beta_{5}$ it meets a translate of $-T$. If we delete the copies of the simplices $\alpha_{5}$ and the tiles $T$ and $-T$ themselves, then the cross-polytopes $\beta_{5}$ are the facets of $J_{6}$; they meet four around each 3 -face $\alpha_{3}$ and form a surface which splits $\mathbb{E}^{6}$ into two congruent parts.

When $r=2$, the dual is an apeirotope with blended vertex-figure. Hence, potentially at least, we can apply the operation $\kappa$. However, this does not yield a genuine regular 6-polytope of full rank; instead, we obtain the first of the two examples which we had to exclude in [16, Sect. 10] as non-polytopes. Indeed, this viewpoint can be made to provide an alternative explanation for its non-polytopality.

Theorem 5.7 prevents us from applying the Petrie operation $\pi$ to the polytopes $J_{n}$, but the halving operation $\eta$ will work. In the present case, $\eta$ is actually invertible (a double application of $\eta$ recovers the original polytope, with a change of generators). When $n=4$ (that is, $r=0$ ), as in [24, Sect. 10E] we obtain the universal polytope

$$
\left\{\left\{4, \frac{4}{1,2} \mid 3\right\},\left\{\frac{4}{1,2}, 4 \mid 3\right\}\right\} \cong\{\{4,4 \mid 3\},\{4,4 \mid 3\}\} ;
$$

it has the same 20 vertices as before (those of $0_{22}$ ). Combinatorially, this polytope is self-dual; however, geometrically it is not, the dual actually being

$$
\left\{\left\{\frac{4}{1,2}, 4 \mid 3\right\},\left\{4, \frac{4}{1,2} \mid 3\right\}\right\} \text {. }
$$

The symmetry between these two polytopes suggests that we may be able to apply $\eta$ to the latter. Indeed we can; perhaps it is not surprising that the result is

$$
\left\{\left\{\frac{4}{1,2}, 4 \mid 3\right\},\left\{4, \frac{4}{1,2} \mid 3\right\}\right\}^{\eta}=\left\{\left\{3, \frac{4}{1,2}\right\},\left\{\frac{4}{1,2}, 4 \mid 3\right\}\right\},
$$

isomorphic to $J_{4}$, but geometrically distinct. In contrast to $J_{4}$, this polytope does not have a geometric dual, as its mirror vector $(3,3,4,3)$ may indicate.

Remark 13.1 The symmetry group of $\left\{\left\{4, \frac{4}{1,2} \mid 3\right\},\left\{\frac{4}{1,2}, 4 \mid 3\right\}\right\}$ has generators

$$
\begin{aligned}
& R_{0}=\left(\begin{array}{ll}
2 & 3
\end{array}\right) \text {, } \\
& R_{1}=-\left(\begin{array}{lll}
0 & 5
\end{array}\right)\left(\begin{array}{ll}
1 & 3
\end{array}\right)\left(\begin{array}{l}
2 \\
2
\end{array}\right), \\
& R_{2}=\text { (4 5), } \\
& R_{3}=-\left(\begin{array}{ll}
0 & 5
\end{array}\right)\left(\begin{array}{ll}
1 & 4
\end{array}\right)\left(\begin{array}{ll}
2 & 3
\end{array}\right) \text {, }
\end{aligned}
$$


working in the hyperplane $\mathbb{H}^{5} \leq \mathbb{E}^{6}$, with the same conventions as before. The mirror vector here is $(4,3,4,3)$, which explains why the geometric dual is different.

We may now apply $\pi$ to both these (isomorphic) polytopes, to obtain

$$
\begin{aligned}
& \left\{\left\{4, \frac{4}{1,2} \mid 3\right\},\left\{\frac{4}{1,2}, 4 \mid 3\right\}\right\}^{\pi}=\left\{\left\{4, \frac{6}{2,3}: \frac{5}{1,2} \mid 3\right\},\left\{\frac{6}{2,3}, 4: \frac{4}{1,2} \mid 3\right\}\right\}, \\
& \left\{\left\{\frac{4}{1,2}, 4 \mid 3\right\},\left\{4, \frac{4}{1,2} \mid 3\right\}\right\}^{\pi}=\left\{\left\{\frac{4}{1,2}, \frac{6}{1,3}: \frac{5}{1,2} \mid 3\right\},\left\{\frac{6}{1,2}, \frac{4}{1,2}: 4 \mid 3\right\}\right\} .
\end{aligned}
$$

These polytopes have no geometric duals. The facets of the Petrials are isomorphic to $\{4,6: 5 \mid 3\}$; their dimensions are 4 and 5 , respectively.

The polytope $\left\{\left\{4, \frac{4}{1,2} \mid 3\right\},\left\{\frac{4}{1,2}, 4 \mid 3\right\}\right\}$ and its Petrial are distinguished (among finite 4-polytopes of dimension 5) in having vertex-figures with mirror vector $(2,3,2)$. As we have already pointed out, the only way to incorporate two automorphisms is to have a diagram with a redundant generator; for the first polytope, we thus have

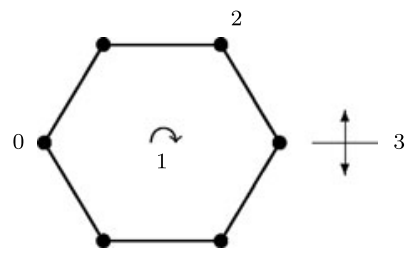

This looks like [24, Fig. 10C1] (which is unfortunately mislabelled), but the latter represents the infinite group, where one of the hyperplane mirrors is displaced from the origin $o$. Working clockwise around the hexagonal diagram from $R_{0}=\left(\begin{array}{l}2 \\ 2\end{array}\right)$, the remaining nodes correspond to (3 5), (4 5), (1 4), (l $\left.\begin{array}{ll}0 & 1\end{array}\right)$ and $\left(\begin{array}{ll}0 & 2\end{array}\right)$; any of these generators can be taken to be the redundant one. After applying $\pi$, the new $R_{1}$ is (1 2)(3 4), which reflects the diagram in a vertical line.

In the cases $r=1,2$, there are further operations, of which $\eta$ is the first. Suppose that $\mathcal{P}$ is an abstract regular $n$-polytope of type $\left\{3^{n-3}, 4, q\right\}$ for some $q$, with group $\boldsymbol{G}=\left\langle\boldsymbol{r}_{0}, \ldots, \boldsymbol{r}_{n-1}\right\rangle$. A simplex dissection result of Debrunner [7] can be interpreted as a mixing operation $\mu_{k}:\left(\boldsymbol{r}_{0}, \ldots, \boldsymbol{r}_{n-1}\right) \mapsto\left(\boldsymbol{s}_{0}, \ldots, \boldsymbol{s}_{n-1}\right)$, where

$$
\boldsymbol{s}_{j}:= \begin{cases}\boldsymbol{r}_{j} & \text { for } j=0, \ldots, k-1, \\ \boldsymbol{r}_{k} \boldsymbol{r}_{k+1} \cdots \boldsymbol{r}_{n-2} \boldsymbol{r}_{n-3} \cdots \boldsymbol{r}_{k} & \text { for } j=k, \\ \boldsymbol{r}_{n+k-j} & \text { for } j=k+1, \ldots, n-1 .\end{cases}
$$

The range is $0 \leq k \leq n-2$; formally, we can take $\mu_{-1}$ to be duality. Observe that $\mu_{n-2}$ is the identity, while $\mu_{n-3}=\eta$. We used the same operation (in a dual form, with different indices) in [15]; see also [24, Sect. 14A]. The new group $\boldsymbol{G}_{k}:=\left\langle\boldsymbol{s}_{0}, \ldots, \boldsymbol{s}_{n-1}\right\rangle$ is a subgroup of $\boldsymbol{G}_{n-2}:=\boldsymbol{G}$ of index a divisor of $\left(\begin{array}{c}n-1 \\ k+1\end{array}\right)$, and the operation $\mu_{k}$ leads (potentially, at least) to a new regular polytope of type $\left\{3^{k-2}, 4, q, q, 4,3^{n-k-3}\right\}$. (It is routine to check that $\boldsymbol{s}_{k-1} \boldsymbol{s}_{k} \sim \boldsymbol{r}_{n-3} \boldsymbol{r}_{n-2}$ and $\boldsymbol{s}_{k} \boldsymbol{s}_{k+1} \sim \boldsymbol{r}_{n-2} \boldsymbol{r}_{n-1}$.)

We now apply the $\mu_{k}$ to the $J_{n}$. With $J_{4}$, the sequence is completed to

$$
\left\{3,4, \frac{4}{1,2}\right\},\left\{4, \frac{4}{1,2}, 4\right\},\left\{\frac{4}{1,2}, 4, \frac{4}{1,2}\right\},\left\{4, \frac{4}{1,2}, 3\right\} ;
$$


the first and last are geometric duals. For $J_{5}$, we obtain the sequence

$$
\left\{3,3,4, \frac{4}{1,2}\right\},\left\{3,4, \frac{4}{1,2}, 4\right\},\left\{4, \frac{4}{1,2}, 4, \frac{4}{1,2}\right\},\left\{\frac{4}{1,2}, 4, \frac{4}{1,2}, 3\right\},\left\{4, \frac{4}{1,2}, 3,3\right\},
$$

the first two being $J_{5}$ and $J_{5}^{\eta}$. From $J_{6}$ we obtain

$$
\begin{aligned}
& \left\{3,3,3,4, \frac{4}{1,2}\right\},\left\{3,3,4, \frac{4}{1,2}, 4\right\},\left\{3,4, \frac{4}{1,2}, 4, \frac{4}{1,2}\right\},\left\{4, \frac{4}{1,2}, 4, \frac{4}{1,2}, 3\right\} \\
& \left\{\frac{4}{1,2}, 4, \frac{4}{1,2}, 3,3\right\},\left\{4, \frac{4}{1,2}, 3,3,3\right\}
\end{aligned}
$$

the first two are $J_{6}$ and $J_{6}^{\eta}$. In all these sequences, we should interpret the sections $\left\{4, \frac{4}{1,2}\right\}$ or $\left\{\frac{4}{1,2}, 4\right\}$ as $\left\{4, \frac{4}{1,2} \mid 3\right\}$ or $\left\{\frac{4}{1,2}, 4 \mid 3\right\}$, respectively. In contrast to the case of $J_{4}$, polytopes (or apeirotopes) symmetric about the middle of the sequences are geometric duals.

We can now apply $\eta$ to those two polytopes with 4 -coface $\left\{\left\{\frac{4}{1,2}, 4 \mid 3\right\},\left\{4, \frac{4}{1,2} \mid 3\right\}\right\}$. We then obtain

$$
\begin{gathered}
\left\{4, \frac{4}{1,2}, 4, \frac{4}{1,2}\right\}^{\eta}=\left\{4,3, \frac{4}{1,2}, 4\right\}, \\
\left\{3,4, \frac{4}{1,2}, 4, \frac{4}{1,2}\right\}^{\eta}=\left\{3,4,3, \frac{4}{1,2}, 4\right\} .
\end{gathered}
$$

In these, $\left\{4,3, \frac{4}{1,2}\right\}=\left\{4,3, \frac{4}{1,2} \mid 3\right\} \cong\{4,3,4\}_{(3,0,0)}$ is a torus; see [22] or [24, Sect. 6D]. The facet of the second polytope (actually apeirotope) is isomorphic to the universal locally toroidal polytope

$$
\mathcal{T}_{(3,0,0)}^{5}=\{\{3,4,3\},\{4,3,4 \mid 3\}\}
$$

of [22] or [24, Sect. 12B]; its facet is the 24-cell. Neither of these has a geometric dual.

Finally, rather than being proper subgroups, all the symmetry groups in each case are isomorphic. Indeed, except for the duals $J_{n}^{\delta}$ of the original polytopes, all in each family have the same vertices.

We cannot apply $\pi$ to any of the polytopes in the sequences in the cases $r=1,2$; we fall foul of Theorem 5.7, because of sections or holes $\{3\}$. We can always apply $\zeta$ to the polytopes in the $J_{4}$ and $J_{5}$ families, and $\kappa$ to the apeirotopes in the $J_{6}$ family. However, as is frequently the case, we obtain nothing of any striking interest; note that applying $\kappa$ will result in infinite facets (or even infinite 2 -faces $\left\{\frac{4}{0,1}\right\}$ from original faces $\left.\left\{\frac{4}{1,2}\right\}\right)$.

\section{Rotational Symmetry Groups}

In dimensions 3 and 4, there are many examples of regular polytopes (or apeirotopes) of nearly full rank whose symmetry groups consist of direct (orientation preserving) isometries only. We shall call such polytopes handed (suitable words of Greek or Latin origin have been pre-empted for other purposes). To be more exact, in $\mathbb{E}^{3}$ we have three handed apeirotopes, while in $\mathbb{E}^{4}$ we have a family of handed polytopes 
which we treated using quaternions, and some sporadic apeirotopes related to them. (Note that a polytope of full rank $n$ cannot be handed, since the last of its generating reflexions $R_{n-1}$ must have a hyperplane mirror-see [16].)

It is natural to ask whether handed examples exist in $\mathbb{E}^{d}$ for $d \geq 5$. In fact, there is one solitary example of a (finite) handed regular 4-polytope in $\mathbb{E}^{5}$, and while there is a closely related polytope in $\mathbb{E}^{5}$ (actually, a double cover), it is not handed. There are no handed regular polytopes or apeirotopes of higher rank.

Before we describe our example, we make some remarks about the general situation.

Proposition 14.1 Let $P$ be a regular polytope (or apeirotope) of nearly full rank, whose vertex-figure $Q$ is handed. If $P$ is not itself handed, then the edge-circuits of $P$ are all even.

Proof This is clear. If $P$ is not handed, then its symmetry $R_{0}$ interchanging the two vertices of the initial edge must be opposite; thus vertex-figures at adjacent vertices have opposite handedness. An odd edge-circuit would then reverse the local orientation of the vertex-figure at the initial vertex, leading to a contradiction.

Our investigation must begin with the (finite) regular 4-polytopes in $\mathbb{E}^{5}$. The potential vertex-figures $Q$ here are the regular polyhedra in $\mathbb{E}^{4}$ with mirror vector $(2,2,2)$; these fall into two classes. The first are those which can be derived by the operation

$$
\varsigma:\left(S_{1}, S_{2}, S_{3}\right) \mapsto\left(S_{2}, S_{1} S_{3},\left(S_{1} S_{2}\right)^{2}\right)=:\left(R_{1}, R_{2}, R_{3}\right)
$$

on the group generators of some other regular polyhedron with square faces. (The indices emphasize that we are thinking of $Q$ as a vertex-figure.) For our purposes, it is crucial to observe (see [23] — as applied to the dual polyhedron) that these are selfPetrie and that the Petrie operation $\pi$ is induced by conjugating by the hyperplane reflexion $S_{1}$. (In this class we also include two closely related handed polyhedra; see the following discussion.) The second class consists of the remainder; we begin by dismissing these.

Recall from [17, Sect. 10] the representation by quaternions of the symmetry group of a regular polyhedron $Q$ in $\mathbb{E}^{4}$ with mirror vector $(2,2,2)$ (for the general background to quaternions, see [10]). A typical symmetry of $Q$ is of the form $\mathbf{x} \mapsto \overline{\mathbf{a}} \mathbf{x b}$, where the $\mathbf{a}$ form the left group $\mathcal{G}_{\mathrm{L}}$ and the $\mathbf{b}$ form the right group $\mathcal{G}_{\mathrm{R}}$, each being a finite subgroup of the group $\mathcal{Q}$ of unit quaternions. Here, $\mathcal{G}_{\mathrm{L}}\left(\mathcal{G}_{\mathrm{R}}\right)$ is the lifting (double cover) of a subgroup $\mathrm{G}_{\mathrm{L}}\left(\mathrm{G}_{\mathrm{R}}\right)$ of $\mathrm{SO}_{3}$ generated by half-turns; the resulting permissible quaternion groups are the binary dihedral group $\mathcal{D}_{n}$ of order $4 n$ (for some even $n$ ), the binary octahedral group $\mathcal{O}$ of order 48 , and the binary icosahedral group $\mathcal{I}$ of order 120 . (The underlying rotation groups of $\mathcal{D}_{n}$ for odd $n$ and the binary tetrahedral group $\mathcal{T}$ are not generated by appropriate half-turns.) As point-sets in $\mathbb{E}^{4}, \mathcal{D}_{n}$ consists of two regular $2 n$-gons in orthogonal planes, $\mathcal{O}$ consists of the vertices of a 24 -cell $\{3,4,3\}$ and its dual, while $\mathcal{I}$ is the vertex-set of a 600 -cell $\{3,3,5\}$.

In the second class of polyhedra, we can take $\mathcal{G}_{R}$ to be $\mathcal{O}$ or $\mathcal{I}$. More to the point, because the only identifications between elements of $\mathcal{G}_{L}$ and $\mathcal{G}_{R}$ are $(\mathbf{a}, \mathbf{b}) \leftrightarrow$ 
$(-\mathbf{a},-\mathbf{b})$, it follows that any initial point $\mathbf{x} \neq \mathbf{0}$ will give rise to at least one left coset of $\mathcal{O}$ or $\mathcal{I}$; regarded as a point-set in $\mathbb{E}^{4}$, such a coset violates the nearest points criterion of Proposition 3.8. In other words, no such polyhedron can be a vertex-figure of a regular 4-polytope in $\mathbb{E}^{5}$.

For the first class, we can employ a trick. As we have observed, the Petrie operation $\pi$ on one of these polyhedra $Q$ is induced by conjugation by $S_{1}$ in the group with generators $\left(S_{1}, S_{2}, S_{3}\right)$ to which $\varsigma$ was applied. Indeed, this is nearly the case even for the dodecahedron $Q=\left\{\frac{5}{1,2}, 3: \frac{10}{1,3}\right\}$ or its Petrial; here, there is an analogous hyperplane reflexion $S_{1}$ which transforms $Q$ into an enantiomorphic copy with the same vertices. Thus, if we reintroduce $S_{1}$ into the new group generators $\left(R_{0}, R_{1}, R_{2}, R_{3}\right)$ of our putative regular polytope $P$, then we obtain a group generated by hyperplane reflexions which acts on the vertices and edges of $P$ (and 2-faces as well, for all but the dodecahedron or its Petrial). Our argument has thus led to the following:

Theorem 14.2 The group of a five-dimensional regular 4-polytope with a handed vertex-figure is a subgroup of a hyperplane reflexion group, possibly with outer automorphisms.

Our analysis now proceeds exactly as in Sect. 8, except that we can eliminate the case $([3] \times[3]) \rtimes \mathcal{C}_{2}$ as well, because here the vertices of the corresponding (handed) polyhedron are those of two triangles in orthogonal planes, and there is no such configuration in the vertices of $0_{22}$. We refer the reader to [17] for more details of this polyhedron. (With group ([4] $\times[4]) \rtimes \mathcal{C}_{2}$ we again have the vertices of $\beta_{4}$ as vertex-figure among the vertices of $\beta_{5}$, with $R_{1}$ fixing the initial vertex as before.)

We are thus left with $[3,3,3]$ as the only candidate for the extended group of the vertex-figure $Q$; moreover, $Q$ must have the same vertices as the truncated 4-simplex $0_{21}$. Thus $Q$ can only be the self-Petrie hemi-dodecahedron $\left\{\frac{5}{1,2}, 3: \frac{5}{1,2}\right\} \cong\{5,3: 5\}$. Finally, the whole polytope $P$ can now only have the vertices of the 5 -cube $\gamma_{5}$ or the half-cube $h \gamma_{5}=1_{21}$, and the extended reflexion group of $P$ must be $[3,3,3,4]$ or $\left[3^{2,1,1}\right]$.

The truncated 4-simplex $0_{21}$ is the vertex-figure of the half-cube $h \gamma_{5}$, and its vertices are those of $\gamma_{5}$ at distance (along edge-paths) 2 or 3 from an initial one. We can take the initial vertex to be $(1,1,1,1,1)$ and express $\mathcal{G}(Q)$ as permutations of coordinates as in [18, Sect. 8]. Indeed, if we adopt the generators $R_{1}, R_{2}, R_{3}$ of $\mathcal{G}(Q)$ from [18], then the initial vertex of $Q$ is $\pm(-1,-1,1,1,1)$.

There are two possible choices-within [3,3,3,4], of course-if $R_{0}$ is to be a direct isometry as well (according to Proposition 14.1); the first gives the generators of $\mathcal{G}(P)$ as

$$
\begin{aligned}
& R_{0}: x \mapsto\left(-\xi_{1},-\xi_{2}, \xi_{3}, \xi_{4}, \xi_{5}\right), \\
& R_{1}: x \mapsto\left(\xi_{5}, \xi_{4}, \xi_{3}, \xi_{2}, \xi_{1}\right), \\
& R_{2}: x \mapsto\left(\xi_{2}, \xi_{1}, \xi_{4}, \xi_{3}, \xi_{5}\right), \\
& R_{3}: x \mapsto\left(\xi_{2}, \xi_{1}, \xi_{3}, \xi_{5}, \xi_{4}\right) .
\end{aligned}
$$


Bear in mind that $R_{0}$ must commute with $R_{2}$ and $R_{3}$. The vertices of $P$ then coincide with those of the half-cube $h \gamma_{5}$. (The other choice for $R_{0}$ is $x \mapsto$ $\left(\xi_{2}, \xi_{1},-\xi_{3},-\xi_{4},-\xi_{5}\right)$, which we shall exclude later.)

It is worth recalling from the discussion and [18] that the Petrie operation $\pi$ on $Q$ (that is, $\pi: R_{1} \mapsto R_{1} R_{3}$, giving $R_{1} R_{3}: x \mapsto\left(\xi_{4}, \xi_{5}, \xi_{3}, \xi_{1}, \xi_{2}\right)$ ) is induced by conjugation under the hyperplane reflexion $T: x \mapsto\left(\xi_{2}, \xi_{1}, \xi_{3}, \xi_{4}, \xi_{5}\right)$; we have seen that we were obliged to allow $T$ to be incorporated into the appropriately enlarged group.

We suppress the details of the working, but it is routine to calculate that the (initial) facet $F$ has planar square faces $\{4\}$, Petrie polygons and 2-zigzags $\left\{\frac{5}{1,2}\right\}$ (these are not congruent) and holes $\left\{\frac{4}{1,2}\right\}$. It has 16 vertices - that is, all of those of $h \gamma_{5}$, so that $P$ is flat - and 20 faces. Looking in the tables of [6] shows that we must have $F=$ $\left\{4, \frac{5}{1,2}: \frac{5}{1,2}\right\} \cong\{4,5: 5\}$, with symmetry group $\mathcal{G}(F)$ of order 160 . Either directly or as a consequence, it then follows that $\mathcal{G}(P)=\left[3^{2,1,1}\right]^{+}$, the rotation group of $h \gamma_{5}$, of order $16 \cdot 5 ! / 2=960$; thus there are $6=960 / 160$ facets in all.

Let us add a little to the description of $F$ and $P$. A typical face of $F$ is a diametral square in a cross-polytope facet $\beta_{4}=h \gamma_{4}$ of $h \gamma_{5}$ (thus this facet really occurs as $1_{11}$ rather than as $\{3,3,4\})$. With each diametral square in a given facet $\beta_{4}$ is associated another disjoint one determined by the remaining four vertices; the 20 faces of $F$ fall into ten pairs of such disjoint diametral squares. Two different copies of $F$ (under $\mathcal{G}(P))$ then meet in the two pairs of disjoint squares in opposite facets of $h \gamma_{5}$, and so in four faces in all.

We finally remark that the self-Petriality of $Q$ (induced, as we have said, under conjugation by the hyperplane reflexion $T$ ) yields an enantiomorphic copy of $P$ with the same vertices, edges and square faces.

It is natural to ask whether our polytope is universal with the given facet and vertex-figure. In fact, it is, as an application of the computer algebra package GAP shows (we thank Barry Monson for providing this information). We should be able to see this by an application of the circuit criterion of [24, Theorem 2F4], but so far this has proved elusive. The basic edge-circuits correspond to the triangular faces of $h \gamma_{5}$, and it is clear that any edge-circuit can be obtained by concatenating such triangles. Thus it is enough to show that a typical triangle-whose group has generators $\left(R_{0} R_{3}, R_{1} R_{2} R_{1}\right)$ - can be obtained by concatenating edge-circuits arising from copies of the facet $\left\{4, \frac{5}{1,2}: \frac{5}{1,2}\right\}$. Notwithstanding the lack of a direct proof of the isomorphism and assuming the elimination of the other choice, we have shown the following:

Theorem 14.3 There is a single handed five-dimensional 4-polytope, namely,

$$
\left\{\left\{4, \frac{5}{1,2}: \frac{5}{1,2}\right\},\left\{\frac{5}{1,2}, 3: \frac{5}{1,2}\right\}\right\} \cong\{\{4,5: 5\},\{5,3: 5\}\} .
$$

There is a closely related polytope in $\mathbb{E}^{5}$. It is not hard to see that an application of $\zeta$ to $\left\{\left\{4, \frac{5}{1,2}: \frac{5}{1,2}\right\},\left\{\frac{5}{1,2}, 3: \frac{5}{1,2}\right\}\right\}$ yields a regular polytope with the 32 vertices of the 5 -cube $\gamma_{5}$; this is no longer handed. It is again flat; the Petrie polygons and 2zigzags $\left\{\frac{5}{1,2}\right\}$ of the original facet $F$ are now expanded to decagons $\left\{\frac{10}{1,3}\right\}$. Though its facets are of type $\left\{\frac{4}{1,2}, \frac{5}{1,2} \mid \frac{4}{1,2}\right\} \cong\{4,5 \mid 4\}$, it is not isomorphic to the universal $\{\{4,5 \mid 4\},\{5,3: 5\}\}$, as we soon see. Its group has order $32 \cdot 60=1920$, and, as with 
the handed polytope, it is self-Petrie. Again referring to the previous discussion, we see that this is the only other regular polytope of nearly full rank with vertex-figure $\left\{\frac{5}{1,2}, 3: \frac{5}{1,2}\right\}$.

We are grateful to Egon Schulte for the following observations about universality. Consider the (abstract) polytope $2^{\mathcal{Q}}$ defined in [24, Sect. 8C], with $\mathcal{Q}=\{5,3: 5\}$. Its facets are isomorphic to $2^{\{5\}}=\{4,5 \mid 4\}$; this latter polyhedron has group order $320=2^{5} \cdot 10$ and doubly covers $\{4,5: 5\}$ (its Petrie polygons are of length 10). Further, by [24, Theorem 8E6], the universal polytope $\left\{2^{\{5\}}, \mathcal{Q}\right\}$ is simply $2^{\mathcal{Q}}$, since $\mathcal{Q}$ is weakly neighbourly (any two vertices of $\mathcal{Q}$ lie in a common facet of $\mathcal{Q}$ ). Hence, our polytope is a quotient of $2^{\mathcal{Q}}$, whose group is $C_{2}^{10} \rtimes A_{5}$ of order $2^{10} \cdot 60$, by a factor $2^{5}$. We have not yet determined the extra relations which must be imposed on the group of $2^{\mathcal{Q}}$ to yield the polytope; we do know that the group relation arising from the lift of the triangle of $P$ (namely, $\left.\left(R_{0} R_{3} \cdot R_{1} R_{2} R_{1}\right)^{6}=I\right)$ is not needed, since it already holds in $2^{\mathcal{Q}}$.

Remark 14.4 It is worth noting that the facet $\left\{\frac{4}{1,2}, \frac{5}{1,2} \mid \frac{4}{1,2}\right\}$ is not the standard (geometric) $2^{\{5\}}$; this latter has planar faces and, indeed, is obtained from the generators of the group of the 5 -cube by the mixing operation $\left(S_{0}, \ldots, S_{4}\right) \mapsto\left(S_{0}, S_{1} S_{3}, S_{2} S_{4}\right)=$ : $\left(R_{0}, R_{1}, R_{2}\right)$.

To complete the list in $\mathbb{E}^{5}$, note that both these polytopes $P$ are vertex-figures of apeirotopes apeir $P$; since a point reflexion in $\mathbb{E}^{5}$ is opposite, neither of these apeirotopes is handed. Their symmetry groups $\mathcal{G}(P)$ preclude their being vertexfigures of any other regular apeirotopes; bear in mind our ability to adjoin suitable hyperplane reflexions to these groups.

Proposition 14.1 tells us that (if we want a handed polytope) we cannot replace the reflexion $R_{0}$ of (14.1) by the initial hyperplane reflexion $R_{0}^{\prime}: x \mapsto$ $\left(-\xi_{2},-\xi_{1}, \xi_{3}, \xi_{4}, \xi_{5}\right)$ of $h \gamma_{5}$. However, it might be thought that $S_{0}:=-R_{0}^{\prime}$ could suffice (with $S_{j}:=R_{j}$ for $j=1,2,3$ ), yielding another, this time handed, regular 4polytope with the 32 vertices of $\gamma_{5}$ and group $[4,3,3,3]^{+}$, the rotation group of $\gamma_{5}$; this is the alternative choice mentioned earlier. In fact, this is not the case. The group $\mathcal{G}=\left\langle S_{0}, \ldots, S_{3}\right\rangle$ is actually isomorphic to $\left[3^{2,1,1}\right]$; all we are doing is replacing each opposite element $G \in\left[3^{2,1,1}\right]$ by $-G$. In fact, just thinking about the putative initial facet $F$, we are introducing an element $-R$ for each reflexion $R$ taking the initial vertex of $F$ into the five vertices of its vertex-figure. It is then not too hard to see that we obtain enough such reflexions $-R$ from $F$ alone to generate the whole group $\mathcal{G}$; the group of $F$ thus contains $S_{3}$, contrary to the intersection property.

We now move on to 5-polytopes in $\mathbb{E}^{6}$ and ask whether $Q=\left\{\left\{4, \frac{5}{1,2}: \frac{5}{1,2}\right\},\left\{\frac{5}{1,2}, 3\right.\right.$ : $\left.\frac{5}{1,2}\right\}$ \} could be the vertex-figure of such a regular polytope $P$. Once again, we introduce the hyperplane reflexion which interchanges the vertex-figure of $Q$ with its Petrial, and conclude that the only possibility arises from the Gosset polytope $2_{21}$, whose vertex-figure is $h \gamma_{5}$. (The only other feasible subgroup relationship is $\left[3^{2,1,1}\right]^{+}<\left[3^{3,1,1}\right]<[3,3,3,3,4]$, which would place the vertices of $P$ among those of the 6-cross-polytope, an obvious impossibility.) Since we already know from Sect. 11 that there is a regular 5-polytope with the same 27 vertices, we might hope that our 4-polytope $Q$ could be the vertex-figure of another regular 5-polytope with 
these vertices. However, this is not to be. Proposition 14.1 again shows that the initial reflexion $S_{0}$ switching the vertices of an initial edge $E$ of $2_{21}$ cannot be adjoined to the group of the putative vertex-figure, because $2_{21}$ has odd edge-circuits. Shifting the previous indices by 1 , it follows that the reflexion $R_{0}$ would have to be the product of $S_{0}$ with a half-turn preserving $E$ and commuting with $R_{2}, R_{3}, R_{4}$; since there is no such half-turn, this possibility must be excluded.

Once again, though, it could be thought feasible to replace $S_{0}$ by $R_{0}:=-S_{0}$, yielding a non-handed six-dimensional regular 5-polytope with the 54 vertices of $2_{21}$ and its reflexion in $o$. However, exactly the same argument we deployed in $\mathbb{E}^{5}$ shows that, just from the initial facet, we would already obtain a group $\mathcal{G} \cong\left[3^{2,2,1}\right]$, with opposite elements $G$ replaced by $-G$ as before (note that they remain opposite).

Let us finally remark that the double cover of $\left\{\left\{4, \frac{5}{1,2}: \frac{5}{1,2}\right\},\left\{\frac{5}{1,2}, 3: \frac{5}{1,2}\right\}\right\}$ of type $\left\{\left\{\frac{4}{1,2}, \frac{5}{1,2} \mid \frac{4}{1,2}\right\},\left\{\frac{5}{1,2}, 3: \frac{5}{1,2}\right\}\right\}$ is even more obviously to be excluded as a possible vertex-figure of a regular 5-polytope in $\mathbb{E}^{6}$.

Recall that the focus of this section is handed regular polytopes, though we have also treated related ones. The conclusion which we gain from the discussion is that handedness - for polytopes of nearly full rank-is a phenomenon of low dimensions; indeed, we have a solitary instance of rank 4 and dimension 5 to add to those in $\mathbb{E}^{3}$ and $\mathbb{E}^{4}$.

\section{Final Remarks}

At this point, we hope that we can claim that our enumeration is complete (we have thought this at several times in the past, only subsequently to find new examples). An important key to the enumeration is the elimination in Sect. 8 of most of the candidates for vertex-figure in rank 4; in addition, even in the case in the previous Sect. 14 of handed polytopes, we saw that the symmetry group of a regular polytope or apeirotope of nearly full rank in five or more dimensions is closely related to a Coxeter (reflexion) group. It is worth repeating that the restriction we noted in Sect. 12 on the kind of diagram which permits a single twist leading to a polytope of nearly full rank plays an important rôle in the classification.

It is natural to ask where one might go from here. In four dimensions, the currently open problem is that of classifying the regular apeirohedra; the obvious analogue in five dimensions concerns the finite regular polyhedra. We have previously indicated that we thought that the former problem could be somewhat hard, and we have little reason to suppose anything different about the latter. The difficulty lies in the increasing freedom as the gap between rank and dimension grows, so that techniques that we have applied hitherto become less and less effective. For instance, there need no longer be any obvious association with Coxeter groups, which we found helpful in this paper; thus the problems posed by the regular apeirohedra and 4-apeirotopes in $\mathbb{E}^{5}$ seem very daunting indeed.

Acknowledgements Egon Schulte read carefully and commented extensively on an earlier draft of this paper. We are very grateful for his considered suggestions; any errors and infelicities remaining are our own responsibility. 


\section{References}

1. Arocha, J.L., Bracho, J., Montejano, L.: Regular projective polyhedra with planar faces, I. Aequ. Math. 59, 55-73 (2000)

2. Bracho, J.: Regular projective polyhedra with planar faces, II. Aequ. Math. 59, 160-176 (2000)

3. Coxeter, H.S.M.: Regular skew polyhedra in 3 and 4 dimensions and their topological analogues. Proc. Lond. Math. Soc. (2) 43, 33-62 (1937). (Reprinted with amendments in Twelve Geometric Essays. Southern Illinois University Press, Carbondale, 1968, pp. 76-105.)

4. Coxeter, H.S.M.: Regular Polytopes, 3rd edn. Dover, New York (1973)

5. Coxeter, H.S.M.: The evolution of Coxeter-Dynkin diagrams. In: Bisztriczky, T., McMullen, P., Schneider, R., Ivić Weiss, A. (eds.) Polytopes: Abstract, Convex and Computational. NATO ASI Series C, vol. 440, pp. 21-42. Kluwer, Dordrecht (1994)

6. Coxeter, H.S.M., Moser, W.O.J.: Generators and Relations for Discrete Groups, 4th edn. Springer, Berlin (1980)

7. Debrunner, H.E.: Dissecting orthoschemes into orthoschemes. Geom. Dedic. 33, 123-152 (1990)

8. Dress, A.W.M.: A combinatorial theory of Grünbaum's new regular polyhedra, I: Grünbaum's new regular polyhedra and their automorphism group. Aequ. Math. 23, 252-265 (1981)

9. Dress, A.W.M.: A combinatorial theory of Grünbaum's new regular polyhedra, II: complete enumeration. Aequ. Math. 29, 222-243 (1985)

10. Du Val, P.: Homographies, Quaternions and Rotations. Oxford University Press, Oxford (1964)

11. Elte, E.L.: The Semiregular Polytopes of the Hyperspaces. Hoitsema, Groningen (1912)

12. Gosset, T.: On the regular and semi-regular figures in space of $n$ dimensions. Messenger Math. 29, 43-48 (1900)

13. Grünbaum, B.: Regular polyhedra—old and new. Aequ. Math. 16, 1-20 (1977)

14. McMullen, P.: Realizations of regular polytopes. Aequ. Math. 37, 38-56 (1989)

15. McMullen, P.: Locally projective regular polytopes. J. Comb. Theory, Ser. A 65, 1-10 (1994)

16. McMullen, P.: Regular polytopes of full rank. Discrete Comput. Geom. 32, 1-35 (2004)

17. McMullen, P.: Four-dimensional regular polyhedra. Discrete Comput. Geom. 38, 355-387 (2007)

18. McMullen, P.: Regular apeirotopes of dimension and rank 4. Discrete Comput. Geom. 42, 224-260 (2009)

19. McMullen, P.: Rigidity of regular polytopes (in preparation)

20. McMullen, P., Schulte, E.: Constructions for regular polytopes. J. Comb. Theory, Ser. A 53, 1-28 (1990)

21. McMullen, P., Schulte, E.: Regular polytopes from twisted Coxeter groups and unitary reflexion groups. Adv. Math. 82, 35-87 (1990)

22. McMullen, P., Schulte, E.: Higher toroidal regular polytopes. Adv. Math. 117, 17-51 (1996)

23. McMullen, P., Schulte, E.: Regular polytopes in ordinary space. Discrete Comput. Geom. 17, 449-478 (1997)

24. McMullen, P., Schulte, E.: Abstract Regular Polytopes. Encyclopedia of Mathematics and its Applications, vol. 92. Cambridge University Press, Cambridge (2002)

25. Pellicer, D., Schulte, E.: Regular polygonal complexes in space, I. Trans. Am. Math. Soc. (to appear) 